Supplementary Information for:

\title{
Selective Reduction of $\mathrm{Nb}$ (V) Species to Promote Molecular Nb/Ta Separation
}

Maxwell H. Furigay, ${ }^{\dagger}$ Subhajyoti Chaudhuri, ${ }^{\ddagger}$ Sean M. Deresh, ${ }^{\dagger}$ Alexander B. Weberg, ${ }^{\dagger}$ Pragati Pandey, ${ }^{\dagger}$ Patrick J. Carroll, ${ }^{\dagger}$ George C. Schatz, ${ }^{\star}, \ddagger$ Eric J. Schelter ${ }^{\star}, \dagger$

†P. Roy and Diana T. Vagelos Laboratories, Department of Chemistry, University of Pennsylvania, 231 South $34^{\text {th }}$ Street, Philadelphia, Pennsylvania 19104, United States

‡ Department of Chemistry and Graduate Program in Applied Physics, Northwestern University, 2145 Sheridan Road, Evanston, Illinois 60208, United States

‘E-mail: g-schatz@northwestern.edu; schelter@sas.upenn.edu

Table of Contents

1. Experimental Section 3

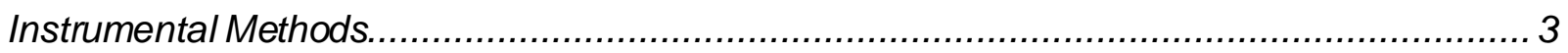

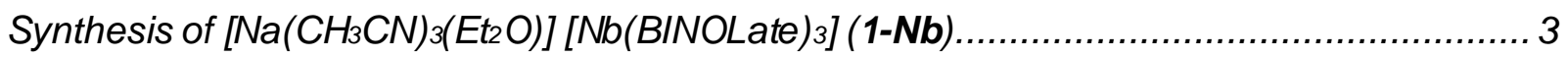

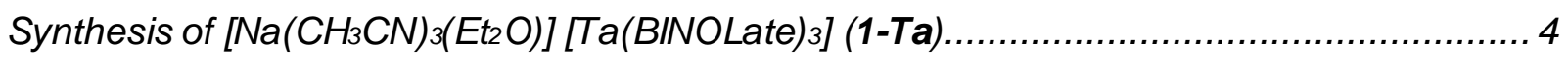

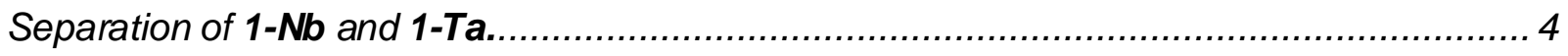

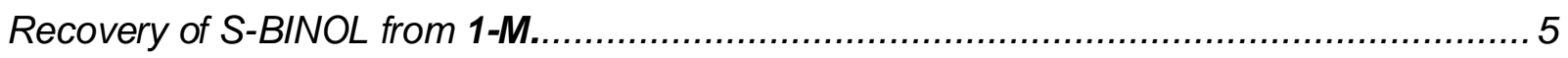

Computational Methods. ........................................................................ 5

Figure S1. Difference in energy for the first and second redox steps between the $\mathrm{Nb}$ and Ta complexes calculated with various levels of theory ..............................................6

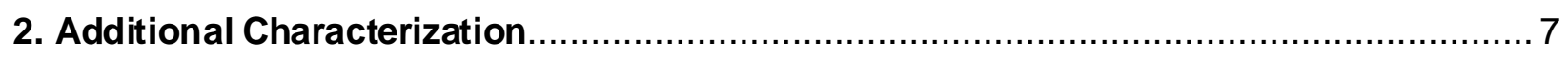

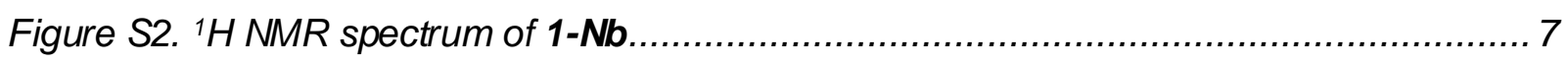

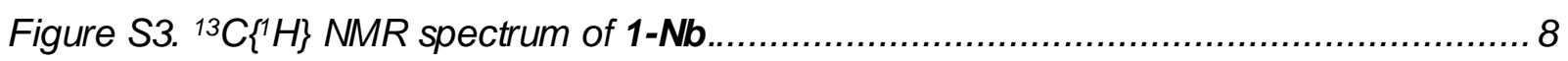

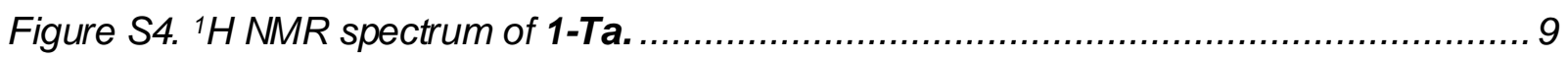

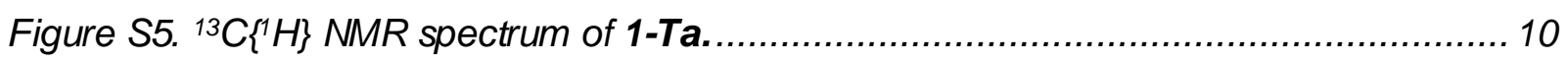

Figure S6. Calculated and experimental UV-Vis spectra for 1-M............................... 11

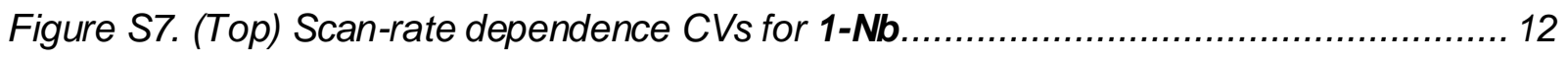

Figure S8. Circular dichroism (CD) data for 1-M............................................ 13

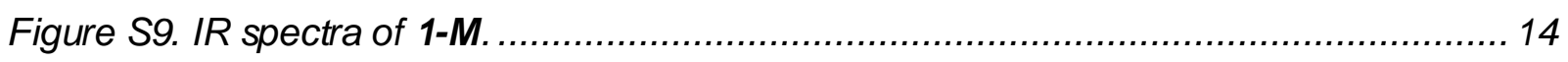

Figure S10. Sample Ta enrichment factor calculations by NMR integrations..................... 15

Table S1. Comparison of ICP vs. NMR Ta enrichment factors, and calculated separations factors by ICP, for three separations trials. ...................................................... 15

Determination of enrichment factors and separation factors ................................... 16 
Figure S11. Sample S-BINOL recovery NMR from 1-Ta................................... 17

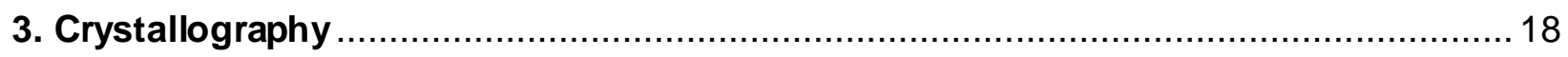

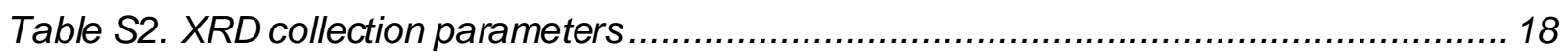

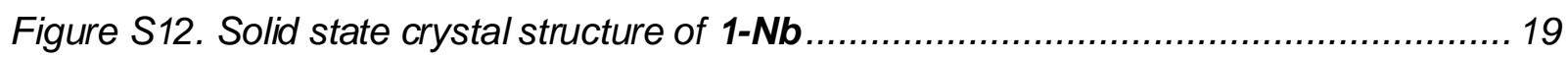

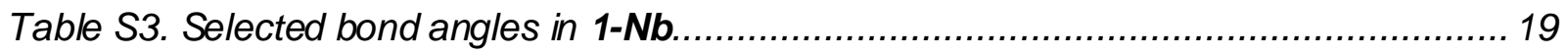

Table S4. Selected bond distances in 1-Nb .................................................... 19

Figure S13. Solid state crystal structure of 1-Ta............................................. 20

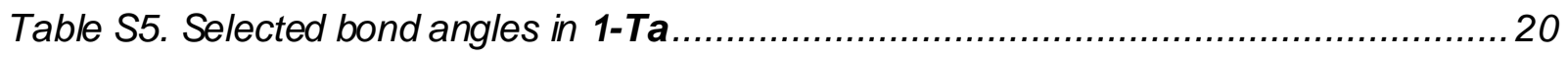

Table S6. Selected bond distances in 1-Ta................................................... 20

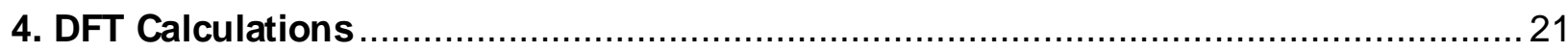

Figure S14. Selected calculated molecular orbitals for 1-M ....................................21

Table S7. XYZ coordinates and Gibbs free energy for the DFT structure of 1-Nb .............22

Table S8. XYZ coordinates and Gibbs free energy for the DFT structure of [1-Nb]- ...........26

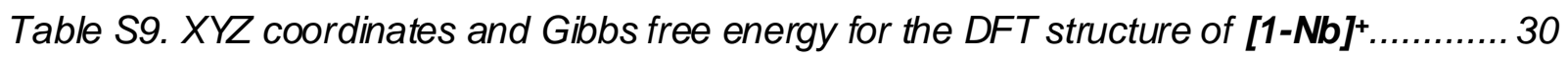

Table S10. XYZ coordinates and Gibbs free energy for the DFT structure of 1-Ta.............. 34

Table S11. XYZ coordinates and Gibbs free energy for the DFT structure of [1-Ta]'........... 38

Table S12. XYZ coordinates and Gibbs free energy for the DFT structure of [1-Ta]+.......... 42 


\section{Experimental Section}

\section{Instrumental Methods}

FT-IR spectra were measured using $\mathrm{KBr}$ pellets on a Bruker Invenior $\mathrm{R}$ spectrometer. ${ }^{1} \mathrm{H}$ and ${ }^{13} \mathrm{C}\left\{{ }^{1} \mathrm{H}\right\}$ NMR spectra were obtained on a Bruker UNI 400 FT NMR spectrometer operating at 400 $\mathrm{MHz}\left({ }^{1} \mathrm{H}\right.$ NMR) or $100 \mathrm{MHz}\left({ }^{13} \mathrm{C}\left\{{ }^{1} \mathrm{H}\right\} \mathrm{NMR}\right)$. Chemical shift values were internally referenced to residual proteo solvent resonances. Cyclic voltammetry (CV) and square wave voltammetry measurements were obtained with a $\mathrm{CH}$ Instruments $620 \mathrm{D}$ potentiostat using a $3 \mathrm{~mm}$ glassy carbon working electrode, a platinum wire counter electrode, and a $\mathrm{Cl}$-doped silver wire pseudoreference electrode. CV measurements were taken with a $0.01 \mathrm{~V}$ sample interval and at $100 \mathrm{mV} / \mathrm{s}$ scan rate unless noted otherwise. Square wave measurements were taken at $0.004 \mathrm{~V}$ increments. All electrochemical measurements were taken in $\mathrm{CH}_{3} \mathrm{CN}$ using $n$-propylammonium tetrakis[3,5bis(trifluoromethyl)phenyl]borate $\left[{ }^{n} \operatorname{Pr}_{4} \mathrm{~N}\right]\left[\mathrm{BAr}_{4}\right]$ electrolyte $(100 \mathrm{mM})$. Freshly sublimed ferrocene (Fc) was used as an internal reference after each data collection session, and all potentials were referenced to the $\mathrm{Fc} / \mathrm{Fc}^{+}$redox couple. Circular dichroism (CD) spectra were recorded using an Aviv Model 425 spectrometer in $\mathrm{CH}_{3} \mathrm{CN}$. UV-Visible (UV-Vis) spectroscopy was obtained on a Perkin Elmer Lambda 950 UV/Vis/NIR spectrometer using $\mathrm{CH}_{3} \mathrm{CN}$ as a solvent. Elemental analyses were performed using a Costech ECS 4010 Analyzer. Inductively Coupled Plasma Optical Emission spectroscopy (ICP-OES) measurements were performed at Galbraith Laboratories, Inc. using a Perkin-Elmer ICP-OES Optima 5300.

\section{Synthetic Methods}

All reactions and manipulations were performed under an inert atmosphere $\left(\mathrm{N}_{2}\right)$ and all glassware was dried in an oven $\left(150^{\circ} \mathrm{C}\right)$ for a minimum of $3 \mathrm{~h}$ prior to use. Degassed solvents were obtained from a commercial solvent purification system consisting of two packed columns of neutral alumina and stored over $3 \AA ̊$ molecular sieves. $\mathrm{M}\left(\mathrm{NMe}_{2}\right)_{5}, \mathrm{NaNH}_{2},(S)-(-)-1,1$ '-bi(2-naphthol) ((S)BINOL), and ( $\left.\mathrm{C}_{5} \mathrm{Me}_{5}\right)_{2} \mathrm{Co}$ were obtained from commercial sources and used without further purification. NMR solvent $\left(\mathrm{CD}_{3} \mathrm{CN}\right)$ was dried over calcium hydride $\left(\mathrm{CaH}_{2}\right)$, distilled under $\mathrm{N}_{2}$, and stored over $3 \AA ̊$ molecular sieves under an inert atmosphere $\left(\mathrm{N}_{2}\right)$.

Synthesis of $\left[\mathrm{Na}\left(\mathrm{CH}_{3} \mathrm{CN}\right)_{3}(\mathrm{Et} 2 \mathrm{O})\right]\left[\mathrm{Nb}(\mathrm{BINOLate})_{3}\right](\mathbf{1 - N b})$.

To a benzene solution (10 mL) of (S)-BINOL (496 mg, $1.73 \mathrm{mmol}, 2.67$ equiv) and $\mathrm{NaNH}_{2}$ (25 mg, $0.65 \mathrm{mmol}, 1.0$ equiv) was added Ta(NMe 2$) 5(203 \mathrm{mg}, 0.648 \mathrm{mmol}, 1.00$ equiv) in benzene $(5 \mathrm{~mL})$ and the mixture was stirred for $24 \mathrm{~h}$ at room temperature. All volatile materials were removed under reduced pressure to yield a colorless solid. The solid was dissolved in minimal $\mathrm{CH}_{3} \mathrm{CN}$ (ca. $5 \mathrm{~mL}$ ) and X-ray-quality crystals of the product were obtained by diffusion of Et2O 
vapor into the $\mathrm{CH}_{3} \mathrm{CN}$ solution of the complex. The crystals were collected and dried under reduced pressure for $1 \mathrm{~h}$ and the product was used without further purification (392 $\mathrm{mg}, 62.4 \%$ yield). IR (cm-1): 3048w, 2966w, 2931w, 2286w, 2291w, 2258w, 1616m, 1588s, 1502m, 1460s, 1426m, 1259m, 1326s, 1269m, 1242s, 1070m, 997m, 948s, 824s, 816s, 745s, 667s, 595s, 559s, 514s. ${ }^{1} \mathrm{H}$ NMR (CD $\left.{ }_{3} \mathrm{CN}, 400 \mathrm{MHz}\right): \delta 7.89$ (d, $\left.12 \mathrm{~Hz}, 6 \mathrm{H}\right), 7.79$ (d, 12 Hz, 6H), 7.67 (d, 12 Hz, 6 H), 7.18 (td, 12 Hz, 4Hz, $6 \mathrm{H}$ ), 7.07-6.92 (m, $12 \mathrm{H}$ ), 3.43 (q, Et2O), 1.96 (s, $\mathrm{CH}_{3} \mathrm{CN}$ ), 1.13 (t, Et2O). ${ }^{13} \mathrm{C}\left\{{ }^{1} \mathrm{H}\right\}$ NMR $\left(\mathrm{CD}_{3} \mathrm{CN}, 100 \mathrm{MHz}\right): \delta 160.96,133.21,129.39,128.26,127.84,125.55,124.98$, 122.57, 121.98, 118.10. Anal. Calc'd for $\left[\mathrm{Na}\left(\mathrm{CH}_{3} \mathrm{CN}\right)_{2}\left(\mathrm{Et}_{2} \mathrm{O}\right)\right]\left[\mathrm{C}_{60} \mathrm{H}_{36} \mathrm{NbO}_{6}\right]: \mathrm{C}, 72.60 \%$; $\mathrm{H}$, 4.66\%; N, 2.49\%. Found C, 72.51\%; H, 4.30\%; N, 2.55\%. (EA samples were dried under reduced pressure for $5 \mathrm{~h}$ prior to analysis, causing the $\mathrm{CH}_{3} \mathrm{CN}$ equiv to change from 3 in the crystal structure to 2 in EA samples. This is consistent with NMRs taken of the EA samples.)

Synthesis of $\left[\mathrm{Na}\left(\mathrm{CH}_{3} \mathrm{CN}\right)_{3}\left(\mathrm{Et}{ }_{2} \mathrm{O}\right)\right]\left[\mathrm{Ta}(\mathrm{BINOLate})_{3}\right](\mathbf{1 - T a})$.

To a benzene solution (10 mL) of (S)-BINOL (388 mg, $1.35 \mathrm{mmol}, 2.75$ equiv) and $\mathrm{NaNH}_{2}$ (21 mg, $0.51 \mathrm{mmol}, 1.0$ equiv) was added Ta(NMe 2$) 5(198 \mathrm{mg}, 0.493 \mathrm{mmol}, 1.00$ equiv) in benzene $(5 \mathrm{~mL})$ and was stirred for $24 \mathrm{~h}$ at room temperature. All volatile materials were removed under reduced pressure to yield a colorless solid. The solid was dissolved in minimal $\mathrm{CH}_{3} \mathrm{CN}$ (ca. $5 \mathrm{~mL}$ ) and X-ray-quality crystals of the product were obtained by diffusion of $\mathrm{Et}_{2} \mathrm{O}$ into the $\mathrm{CH}_{3} \mathrm{CN}$ solution of the complex. The crystals were collected and dried under reduced pressure for $1 \mathrm{~h}$ and the product was used without further purification ( $461 \mathrm{mg}, 88.4 \%$ yield). IR $\left(\mathrm{cm}^{-1}\right): 3052 \mathrm{w}, 2972 \mathrm{w}$, 2929w, 2876w, 2362w, 2292w, 2260w,1616m, 1590s, 1502s, 1463s, 1245s, 1357s, 1330s, 1271s, 1244s, 1069s, 1069s, 998m, 952s, 825s, 817s, 744s, 667s, 598s, 559s, 538w, 505m. 1H $\operatorname{NMR}\left(\mathrm{CD}_{3} \mathrm{CN}, 400 \mathrm{MHz}, 300 \mathrm{~K}\right): \delta 7.94(\mathrm{~d}, 12 \mathrm{~Hz}, 6 \mathrm{H}), 7.81(\mathrm{~d}, 12 \mathrm{~Hz}, 6 \mathrm{H}), 7.69(\mathrm{~d}, 12 \mathrm{~Hz}, 6 \mathrm{H})$, 7.16 (td, 12 Hz, 4Hz, 6 H), 7.02 (td, 12 Hz, 4Hz, 6 H), 6.94 (d, 12 Hz, 6H), 3.43 (q, Et $2 \mathrm{O}$ ), 1.96 (s, $\left.\mathrm{CH}_{3} \mathrm{CN}\right), 1.13$ (t, Et2O). ${ }^{13} \mathbf{C}\left\{{ }^{1} \mathrm{H}\right\}$ NMR $\left(\mathrm{CD}_{3} \mathrm{CN}, 100 \mathrm{MHz}\right): \delta 158.98,133.22,129.31,128.33$, 127.77, 125.59, 124.94, 122.55, 122.46, 118.26. Anal. Calc'd for $\left[\mathrm{Na}\left(\mathrm{CH}_{3} \mathrm{CN}\right)_{2}\left(\mathrm{Et}_{2} \mathrm{O}\right)\right]\left[\mathrm{C}_{6} \mathrm{H}_{36} \mathrm{O} 6 \mathrm{Ta}\right]$ : C, 67.33\%; H, 4.32\%; N, 2.31\%. Found C, 67.79\%; H, 4.09\%; $\mathrm{N}, 2.18 \%$. (EA samples were dried under reduced pressure for $5 \mathrm{~h}$ before analysis, causing the $\mathrm{CH}_{3} \mathrm{CN}$ equiv to change from 3 in the crystal structure to 2 in EA samples. This is consistent with NMRs taken of the EA samples.)

\section{Separation of 1-Nb and 1-Ta.}

A mixture of 1- $\mathrm{Nb}$ (51 mg, $0.043 \mathrm{mmol}, 1.0$ equiv) and 1-Ta (54.1 mg, $0.043 \mathrm{mmol}, 1.0$ equiv) was dissolved in $\mathrm{CH}_{3} \mathrm{CN}(10 \mathrm{~mL})$. An initial aliquot of $1 \mathrm{~mL}$ of the homogenized solution was withdrawn for NMR and ICP analysis. To the remaining solution, ( $\left.\mathrm{C}_{5} \mathrm{Me}_{5}\right)_{2} \mathrm{Co}(141 \mathrm{mg}, 0.428 \mathrm{mmol}, 9.95$ 
equiv) was added and the solution was stirred for $1 \mathrm{~h}$. Volatile materials were removed under reduced pressure to obtain a brown solid. The solid was washed with 3 portions of $10 \mathrm{~mL} \mathrm{Et} 2 \mathrm{O}$, then both the filtrate and filtrand were dried under reduced pressure to yield a dark brown filtrate and a light yellow filtrand, both of which were then analyzed by ICP to determine relevant separation factors.

Recovery of S-BINOL from 1-M.

1-Nb (17.1 mg, $0.015 \mathrm{mmol})$ and 1-Ta $(70.0 \mathrm{mg}, 0.057 \mathrm{mmol})$ were individually dissolved in $\mathrm{CH}_{3} \mathrm{CN}(5 \mathrm{~mL})$ and water $(10 \mathrm{~mL})$ was added (open to air). The reaction mixtures were heated to $60^{\circ} \mathrm{C}$ for $18 \mathrm{~h}$, during which a white precipitate was observed to form. The resulting slurries were filtered, and all volatiles were removed from the filtrate fractions under reduced pressure to y ield white solids. ${ }^{1} \mathrm{H}$ NMR spectra confirmed these materials to be S-BINOL, the purity of which was estimated at $98 \%$ through use of an internal standard (1,3,5-trimethoxybenzene) (Figure S11). Spiking the NMR samples with pure $S$-BINOL further confirmed the composition of these solids (Figure S11). The percent mass recoveries of S-BINOL from 1-M were found to be $97 \%$ (12.3 mg, $0.043 \mathrm{mmol}$ ) for $\mathbf{1 - N b}$ and $96 \%$ (46.9 $\mathrm{mg}, 0.164 \mathrm{mmol}$ ) for $\mathbf{1 - T a}$.

\section{Computational Methods.}

Geometry Optimization: DFT calculations were performed in Orca 4.2 using Becke's threeparameter B3LYP exchange-correlation functional (unrestricted for all the open shell systems) and using the Def2-SVP basis set with an auxiliary Def2/J basis set. All calculations were done in a CPCM acetonitrile solvent (dielectric constant of 36.6).

Electrochemistry: Redox potentials were calculated using energy differences from single point calculations on the optimized structures. Energies were calculated using various methods (Fig. S1) for comparison. No significant difference was observed for the 1-M to [1-M]+ step between the Ta and Nb complexes. However, a noticeable difference was seen in the [1-M]- to 1-M step. For ease of comparison with the cyclic voltammetry experiments, the redox potentials were adjusted by the redox energy corresponding to the $\mathrm{Fc} / \mathrm{Fc}^{+}$couple calculated at the same level of theory. The result is shown in Figure $3(\mathrm{C})$. 


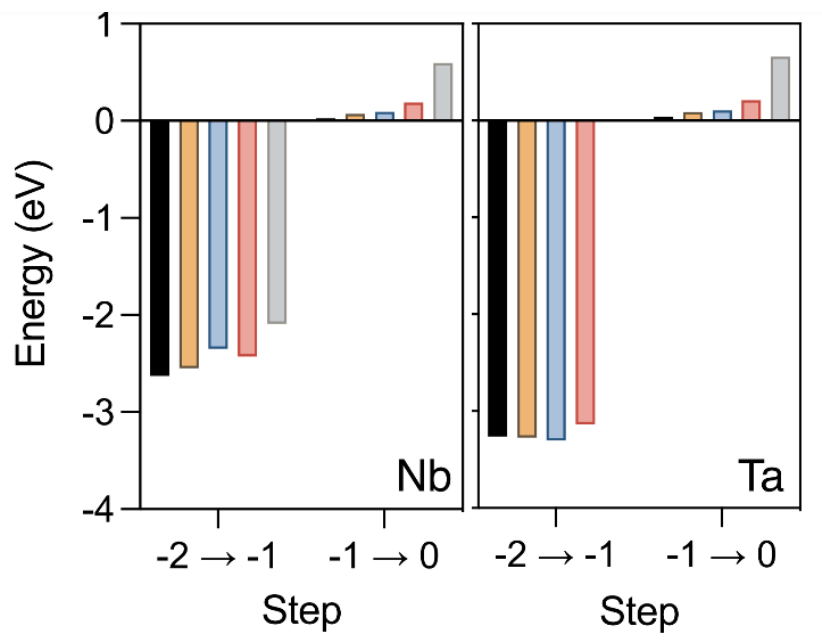

B3LYP / Def2-SVP

B3LYP / Def2-TZVP

B3LYP / ZORA-Def2-TZVP

M06-2X/Def2-SVP

Experiment

Figure S1. Difference in energy for the first and second redox steps between the $\mathrm{Nb}$ and $\mathrm{Ta}$ complexes calculated with various levels of theory. Note that the $\left[\mathrm{Na}\left(\mathrm{CH}_{3} \mathrm{CN}\right)_{3}(\mathrm{DME})\right]^{+}$ counterion was removed for these calculations, so the -2 à -1 step refers to the [1-M] à 1-M redox event, and the -1 à -0 step refers to the $1-\mathbf{M}$ à [1-M]+ redox event.

TD-DFT Calculations: All TD-DFT (with Tamm-Dankoff approximation) calculations were performed using the B3LYP functional with Def2-SVP basis set with an auxiliary SARC/J basis in acetonitrile continuum solvent (dielectric constant of 36.6). Resolution of Identity (RI-J) approximation was used for faster calculation of the Coulomb integrals. For both [1-Nb] ${ }^{-1}$ and [1Ta] $^{-1}$ complexes, the first 300 excited states were calculated. 


\section{Additional Characterization}
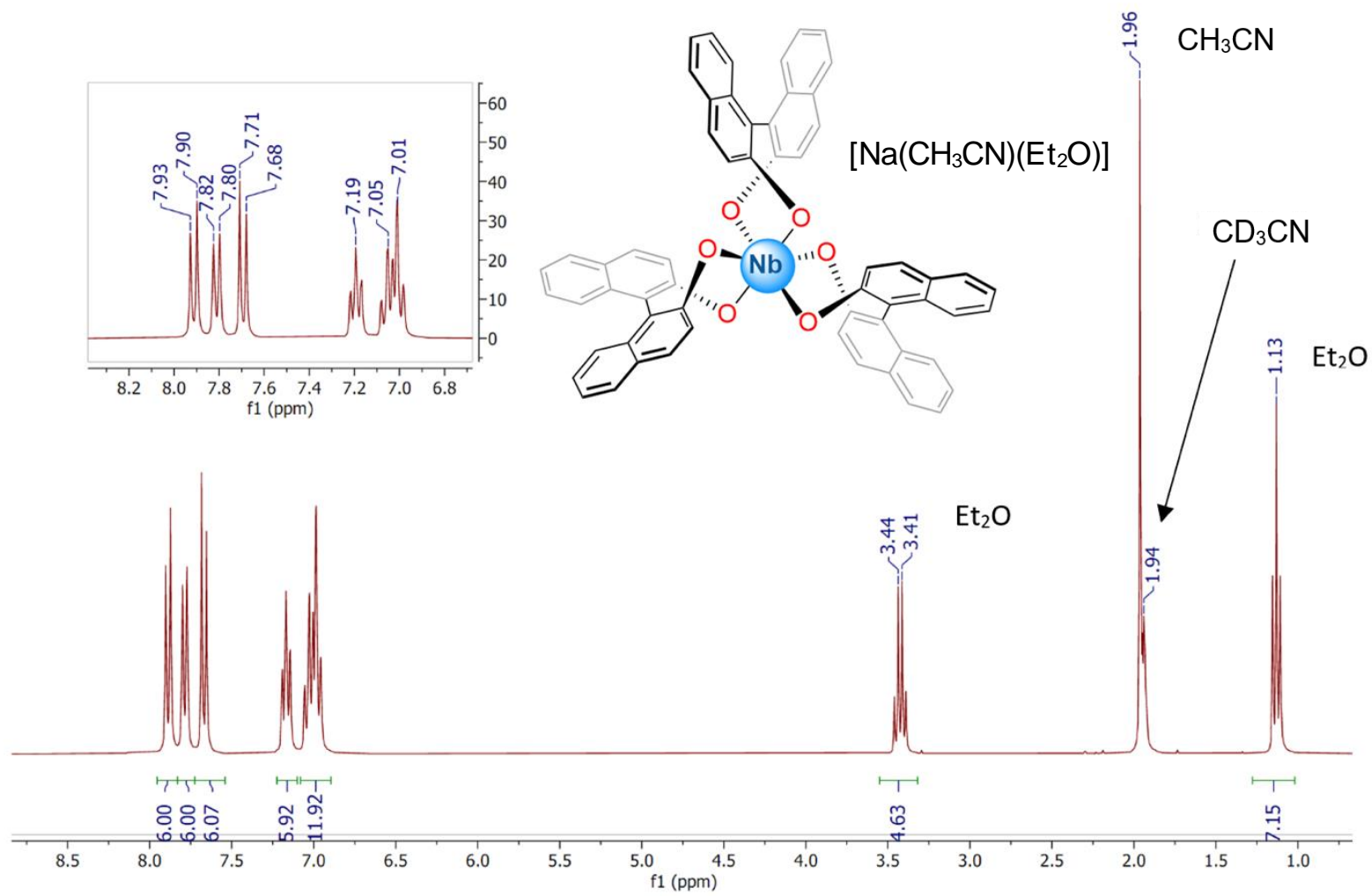

Figure S2. ${ }^{1} \mathrm{H}$ NMR spectrum of $\mathbf{1 - N b}$. 


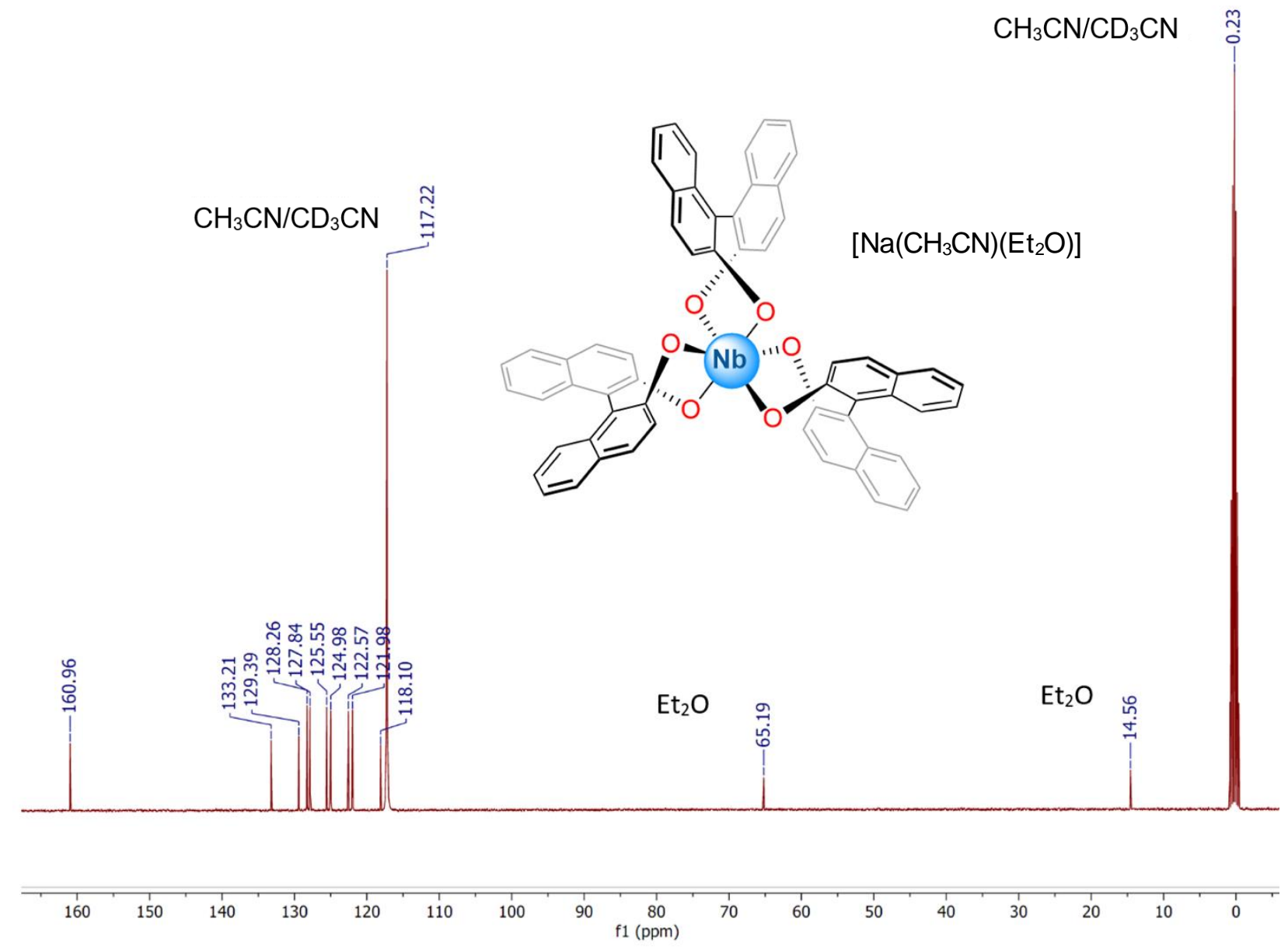

Figure S3. ${ }^{13} \mathrm{C}\left\{{ }^{1} \mathrm{H}\right\}$ NMR spectrum of $\mathbf{1 - N b}$. 


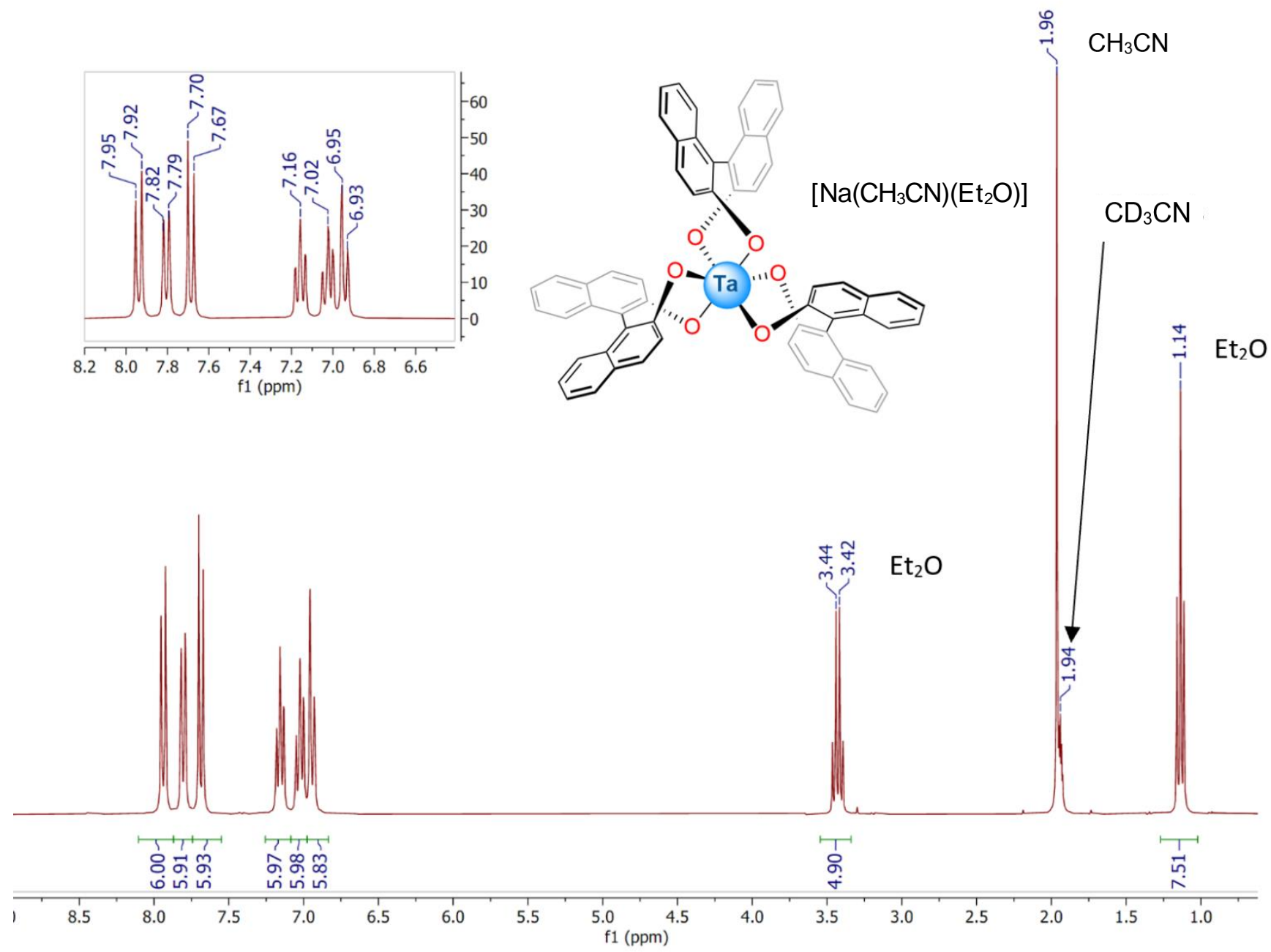

Figure S4. ${ }^{1} \mathrm{H}$ NMR spectrum of 1-Ta. 

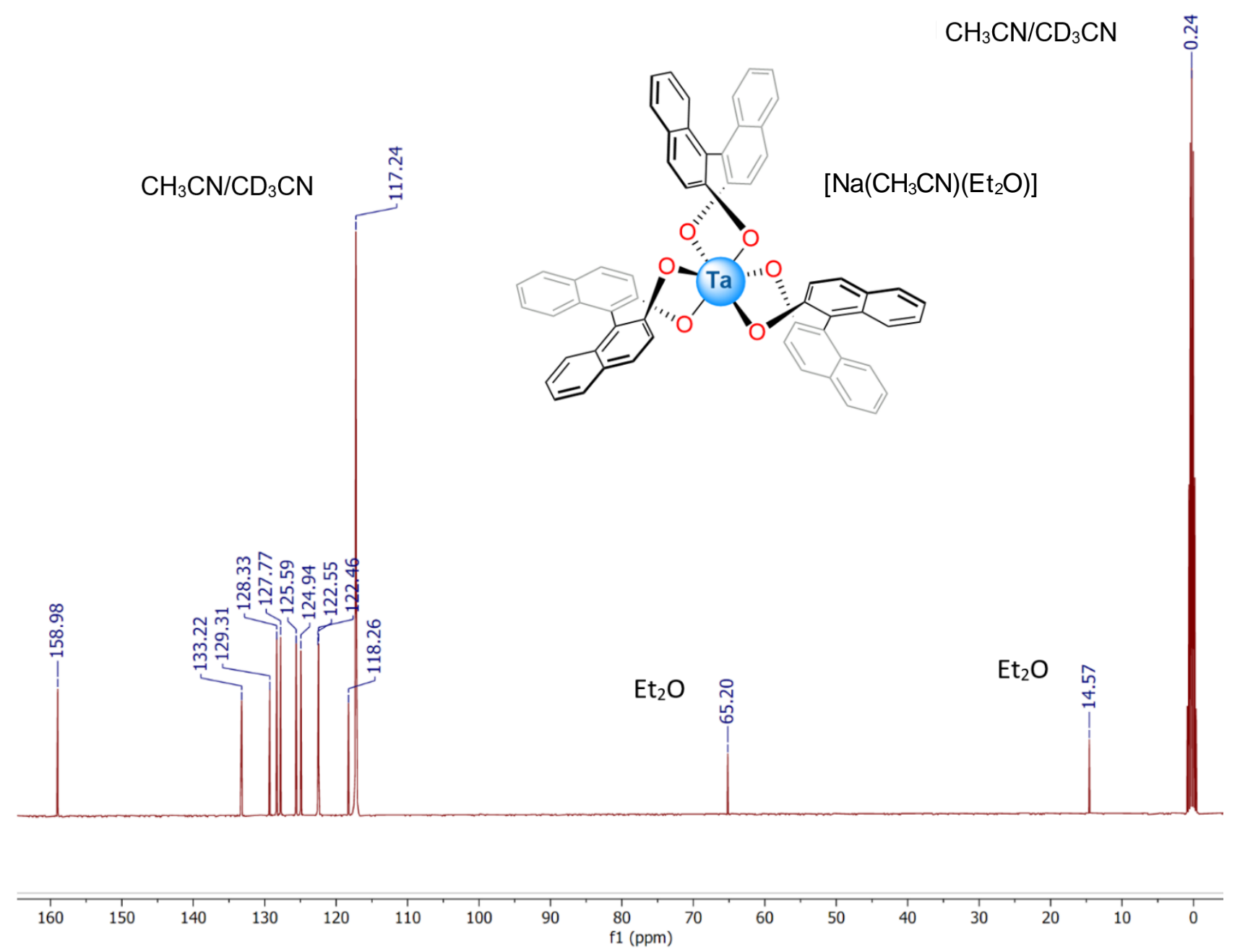

Figure $\mathrm{S} 5 .{ }^{13} \mathrm{C}\left\{{ }^{1} \mathrm{H}\right\}$ NMR spectrum of 1-Ta. 


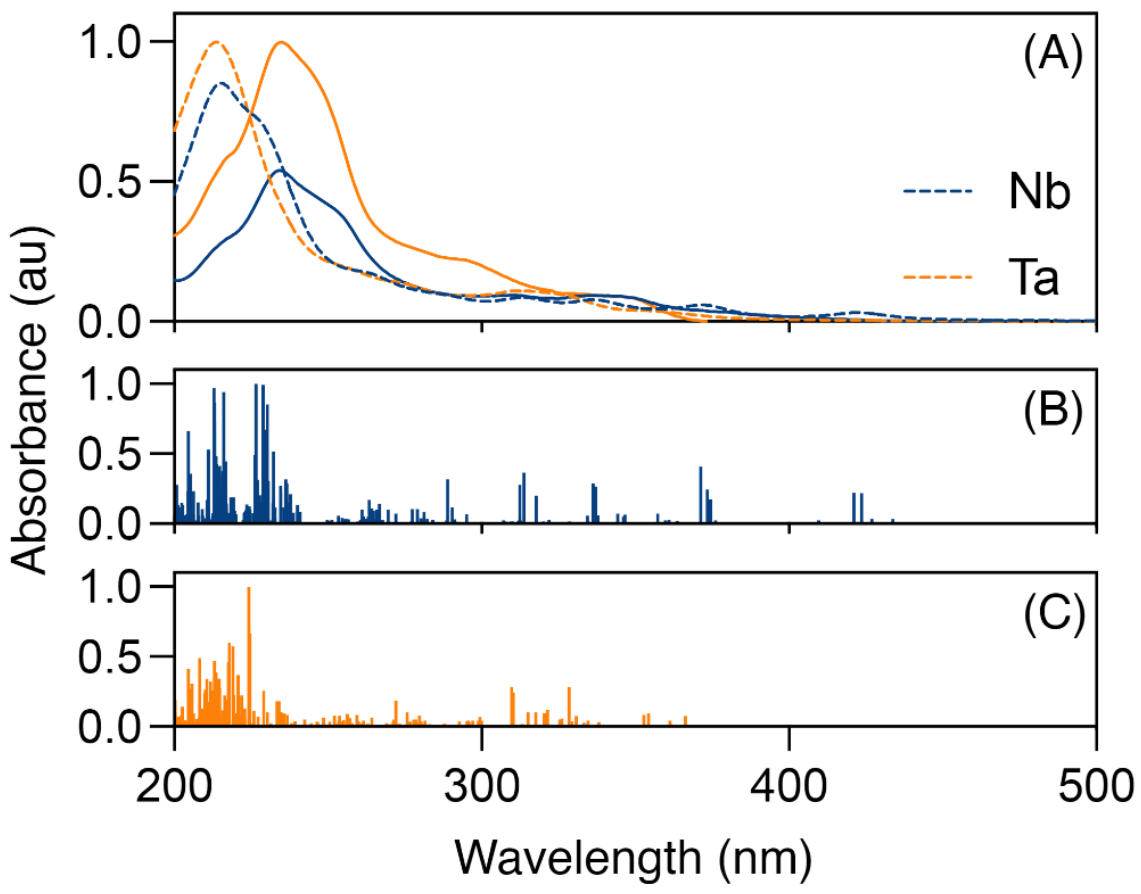

Figure S6. (A) calculated (dashed) and experimental (solid) UV-Vis spectra of 1-M in $\mathrm{CH}_{3} \mathrm{CN}_{\text {. }}$ Calculated UV-Vis stick plots of vertical excitations for 1-Nb (B) and 1-Ta (C). 

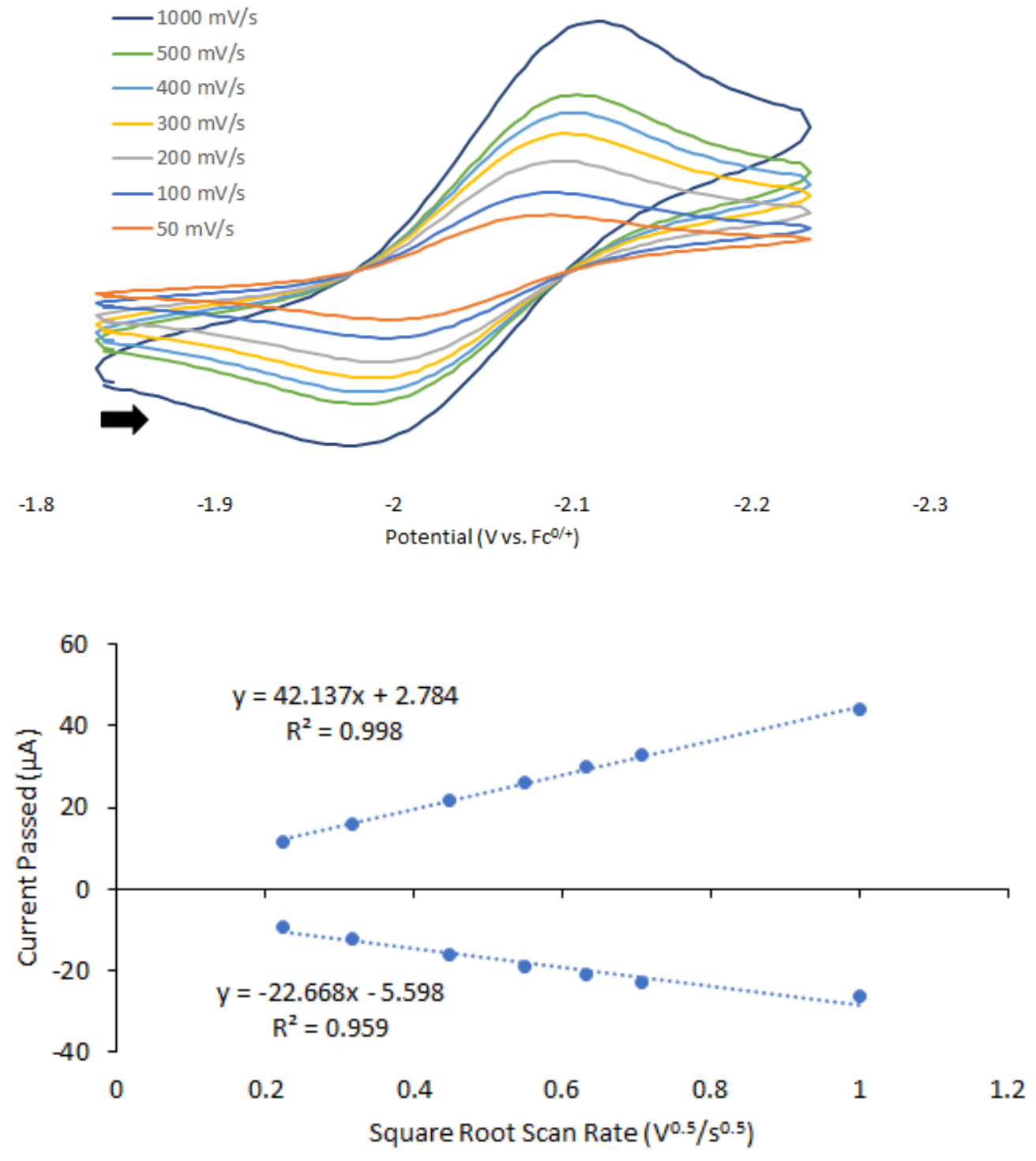

Figure S7. (Top) Scan-rate dependence CVs for $1-\mathrm{Nb}$. CVs were taken in $\mathrm{CH}_{3} \mathrm{CN}$ at $5 \mathrm{mM}$ analyte concentration and with $n$-propylammonium tetrakis[3,5-bis(trifluoromethyl)phenyl]borate) [ $\left.{ }^{\mathrm{P}} \mathrm{Pr}_{4} \mathrm{~N}\right][\mathrm{BArF} 4]$ as the electrolyte at $100 \mathrm{mM}$ concentration. (Bottom) Plot of the total current passed in the redox event depicted above versus the scan rate with linear lines of best fit for the oxidation and reduction waves. 


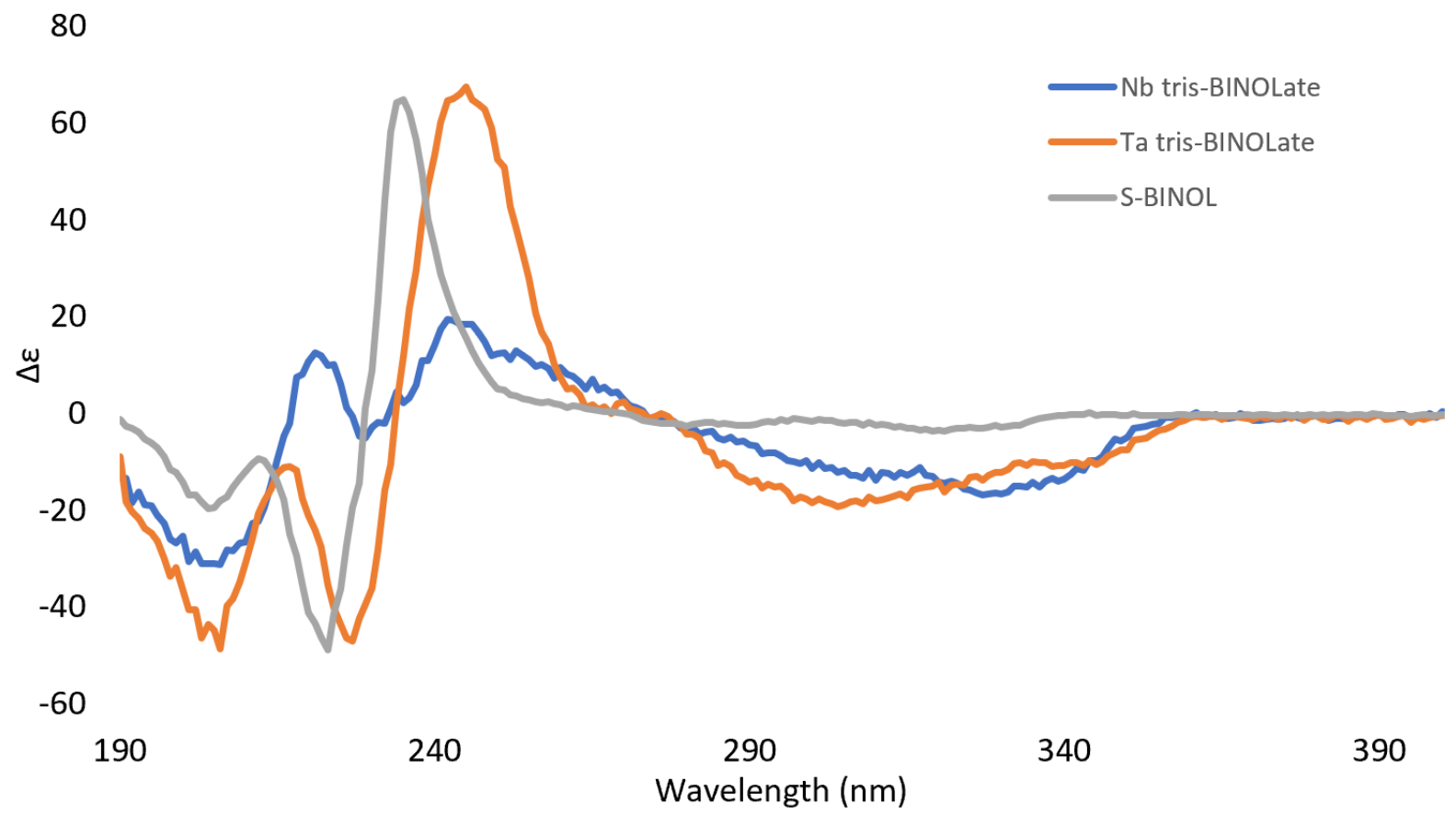

Figure S8. Circular dichroism (CD) data for 1-M and the free ligand (S)-BINOL. Measurements were taken at $0.001 \mathrm{mM}$ analyte concentration in $\mathrm{CH}_{3} \mathrm{CN}$. No $\mathrm{CD}$ response was observed between 400-950 $\mathrm{nm}$. 


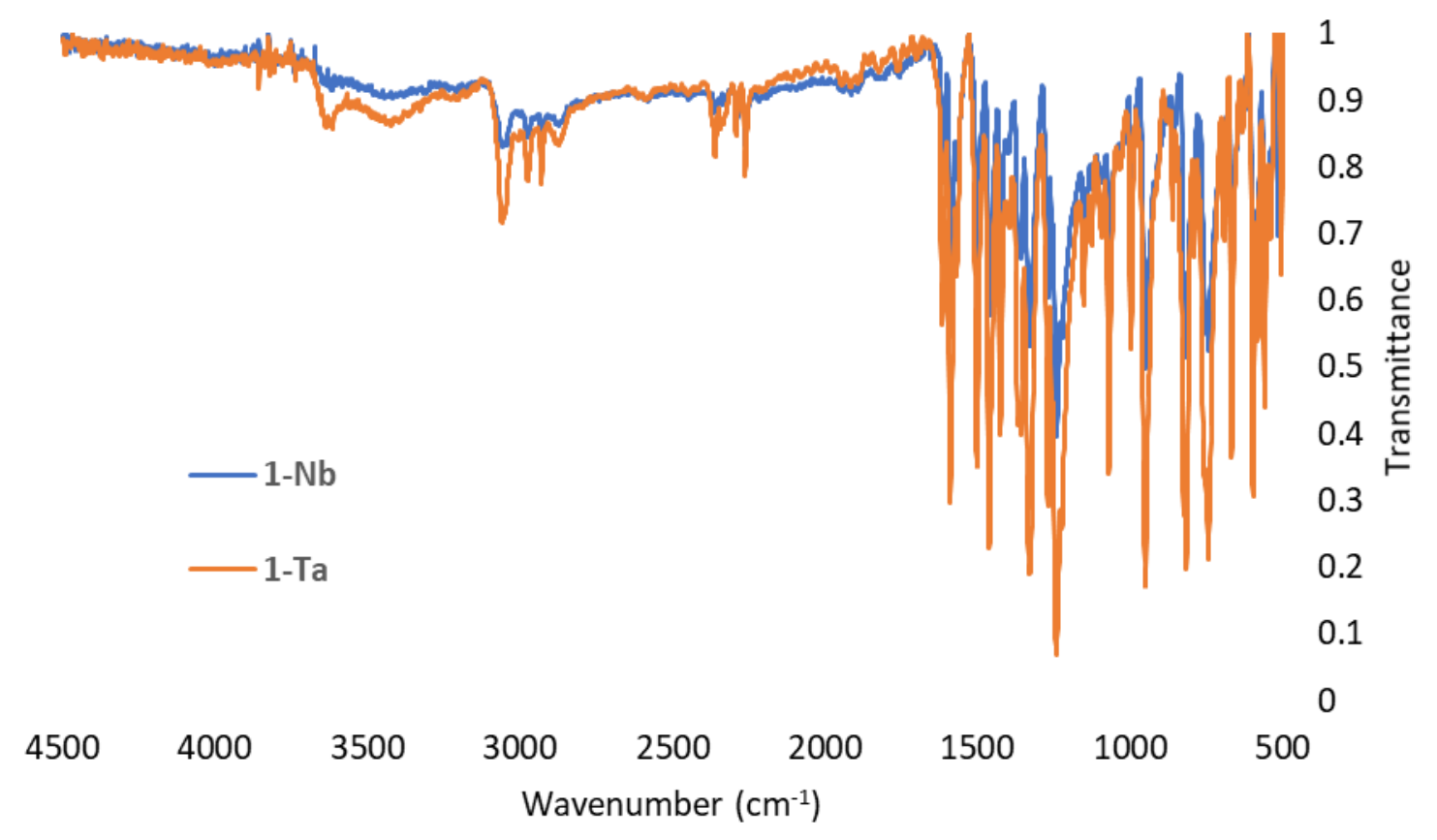

Figure S9. IR spectra of 1-M. IR collected on $\mathrm{KBr}$ pellets. 


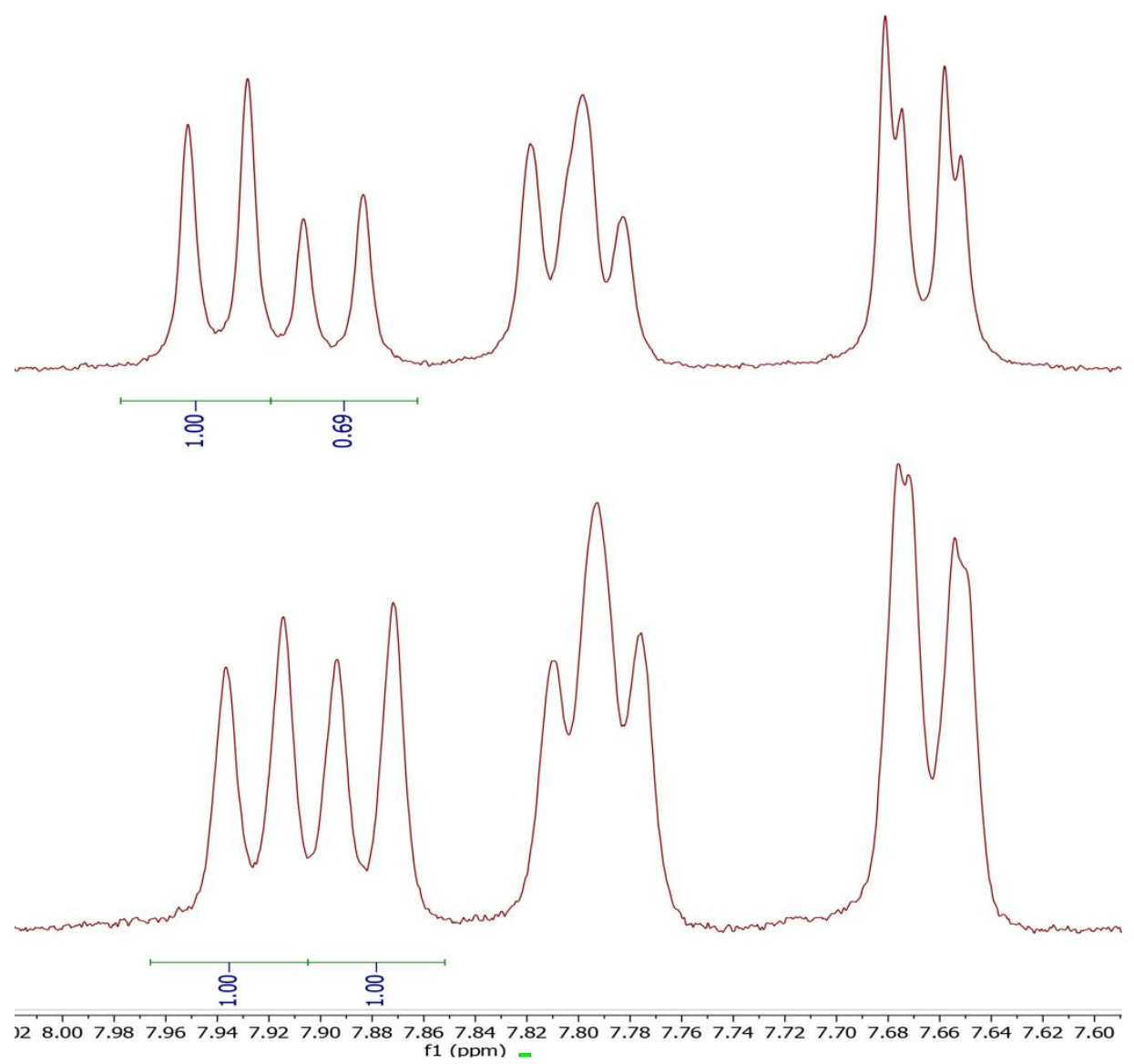

Figure S10. Sample Ta enrichment factor calculations by NMR integrations. (Bottom) Homogenized 1-M before $\mathrm{Co}\left(\mathrm{Cp}^{*}\right)_{2}$ reduction, roughly 1:1 Ta:Nb by NMR integration. (Top) Unreacted 1-M filtrand after reduction and $\mathrm{Et}_{2} \mathrm{O}$ wash, roughly 1:0.69 Ta:Nb by NMR integration. These spectra correspond to the second entry in Table S1.

Table S1. Comparison of ICP vs. NMR Ta enrichment factors, and calculated separations factors by ICP, for three separations trials.

\begin{tabular}{cccccc}
\hline $\begin{array}{c}\text { Init Ta EF } \\
\text { (ICP) }\end{array}$ & $\begin{array}{c}\text { Init Ta EF } \\
\text { (NMR) }\end{array}$ & $\begin{array}{c}\text { Filtrand Ta } \\
\text { EF (ICP) }\end{array}$ & $\begin{array}{c}\text { Filtrand Ta EF } \\
\text { (NMR) }\end{array}$ & $\begin{array}{c}\text { Filtrate Nb EF } \\
\text { (ICP) }\end{array}$ & SF \\
\hline 1.07 & 0.82 & 1.47 & 1.28 & 3.04 & 4.49 \\
\hline 0.96 & 1.00 & 1.70 & 1.45 & 5.11 & 8.66 \\
\hline 1.23 & 1.08 & 1.37 & 1.22 & 4.43 & 6.04 \\
\hline
\end{tabular}




\section{Determination of Enrichment Factors and Separation Factors}

Enrichment factors were calculated using the following equations, where EF represents the enrichment factor and $\mu$ represents millimoles:

$$
\begin{aligned}
\mathrm{EF}_{\mathrm{Nb}, \text { filtrate }} & =* \frac{\mu_{\mathrm{Nb}, \text { filtrate }}}{\mu_{\mathrm{Ta}, \text { filtrate }}} \\
\mathrm{EF}_{\mathrm{Ta}, \text { filtrand }} & =* \frac{\mu_{\mathrm{Ta}, \text { filtrand }}}{\mu_{\mathrm{Nb}, \text { filtrand }}}
\end{aligned}
$$

Separation factors were calculated using the following set of equations, where $D$ represents the diffusion coefficient of a given phase and $S$ represents the separation factor:

$$
\begin{gathered}
D_{M}=* \frac{\mu_{\mathrm{M}, \text { filtrate }}}{\mu_{\mathrm{M}, \text { filtrand }}} \\
\mathrm{S}_{\mathrm{Nb} / \mathrm{Ta}}=\frac{\mathrm{D}_{\mathrm{Nb}}}{\mathrm{D}_{\mathrm{Ta}}}=\frac{\frac{\mu_{\mathrm{Nb}, \text { filtrate }}}{\mu_{\mathrm{Nb}, \text { filtrand }}}}{\frac{\mu_{\mathrm{Ta}, \text { filtrate }}}{\mu_{\mathrm{Ta}, \text { filtrand }}}}=\frac{\mu_{\mathrm{Nb}, \text { filtrate }}}{\mu_{\mathrm{Nb}, \text { filtrand }}} * \frac{\mu_{\mathrm{Ta}, \text { filtrand }}}{\mu_{\mathrm{Ta}, \text { filtrate }}}=\mathrm{EF}_{\mathrm{Nb}, \text { filtrate }} * \mathrm{EF}_{\mathrm{Ta}, \text { filtrand }}
\end{gathered}
$$

The enrichment factors and separation factors were calculated using ICP-OES spectroscopy. Sample calculations are shown below. The initial metal ratio of each homogenized mixture was analyzed by ICP by withdrawing an aliquot of the mixture before treatment with reductant (see SI page 4 for details).

\begin{tabular}{|c|c|c|c|}
\hline & Initial & Filtrand & Filtrate \\
\hline$\mu \mathrm{Ta}$ & 0.336 & 0.459 & 0.0133 \\
\hline$\mu \mathrm{Nb}$ & 0.349 & 0.336 & 0.0587 \\
$\mathrm{EF}_{\mathrm{Nb}, \text { initial }}$ & $=\frac{\mu_{\mathrm{Nb} \text {,initial }}}{\mu_{\mathrm{Ta}, \text { initial }}}=\frac{0.349}{0.336}=1.04$ \\
$\mathrm{EF}_{\mathrm{Nb} \text {,filtrate }}$ & $=\frac{\mu_{\mathrm{Nb}, \text { filtrate }}}{\mu_{\mathrm{Ta}, \text { filtrate }}}=\frac{0.0587}{0.0133}=4.41$ \\
$\mathrm{EF}_{\mathrm{Ta}, \text { filtrand }}$ & $=\frac{\mu_{\mathrm{Ta}, \text { filtrand }}}{\mu_{\mathrm{Nb}, \text { filtrand }}}=\frac{0.459}{0.336}=1.37$
\end{tabular}

$$
\begin{aligned}
\mathrm{S}_{\mathrm{Nb} / \mathrm{Ta}}=\frac{\mathrm{D}_{\mathrm{Nb}}}{\mathrm{D}_{\mathrm{Ta}}} & =\frac{\frac{\mu_{\mathrm{Nb}, \text { filtrate }}}{\mu_{\mathrm{Nb}, \text { filtrand }}}}{\frac{\mu_{\mathrm{Ta}, \text { filtrate }}}{\mu_{\mathrm{Ta}, \text { filtrand }}}}=\frac{\mu_{\mathrm{Nb}, \text { filtrate }}}{\mu_{\mathrm{Nb}, \text { filtrand }}} * \frac{\mu_{\mathrm{Ta}, \text { filtrand }}}{\mu_{\mathrm{Ta}, \text { filtrate }}}=\mathrm{EF}_{\mathrm{Nb}, \text { filtrate }} * \mathrm{EF}_{\mathrm{Ta}, \text { filtrand }}=4.41 * 1.37 \\
& =6.04
\end{aligned}
$$




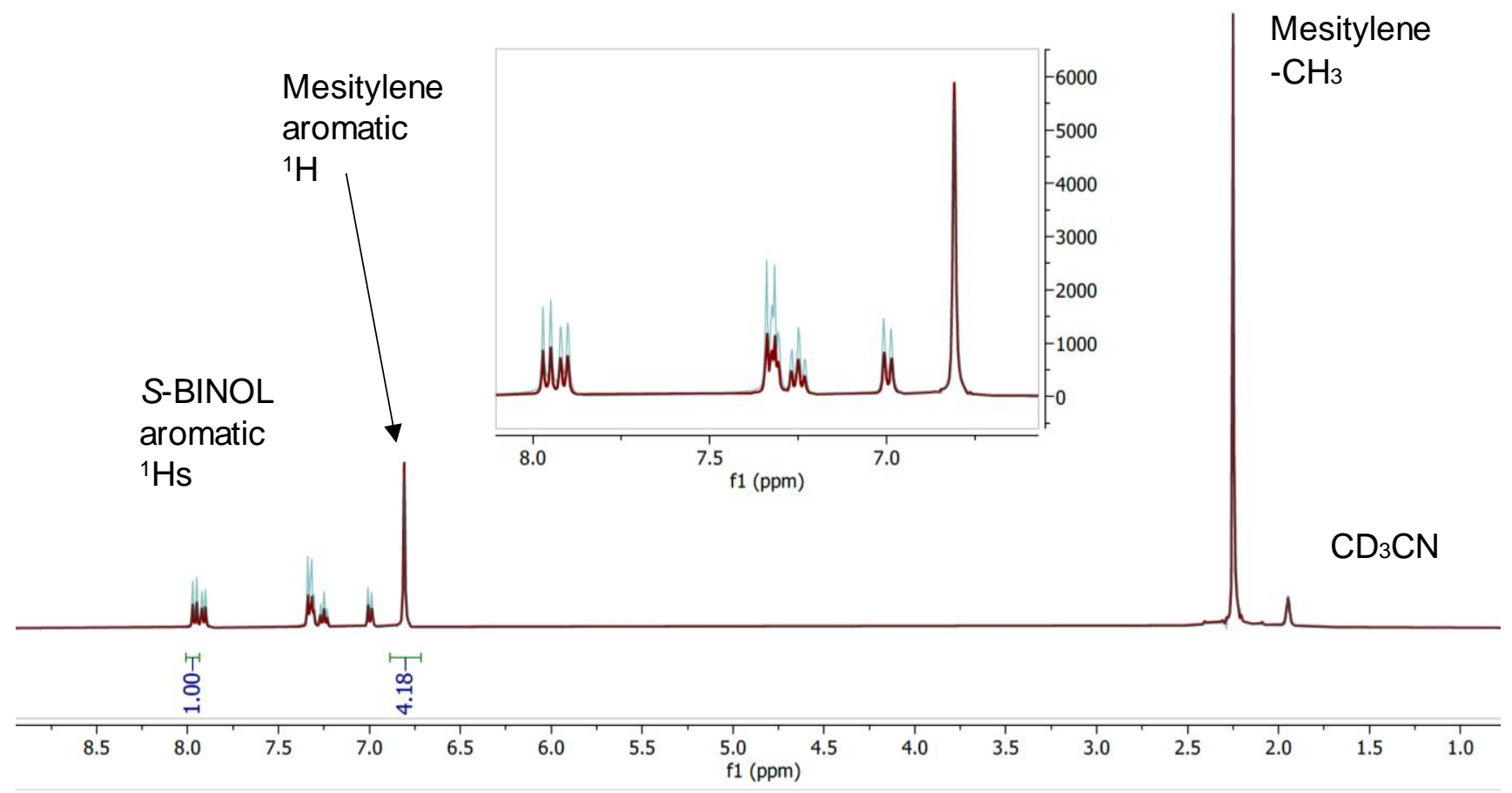

Figure S11. Sample S-BINOL recovery NMR from 1-Ta with 1,3,5-trimethylbenzene (mesitylene) internal standard added for quantification purposes, before (red) and after (green) additional S-BINOL was spiked in. 


\section{Crystallography}

Instrumentation. X-ray intensity data were collected on a Rigaku XtaLAB Synergy-S

diffractometer ${ }^{1}$ equipped with an HPC area detector (Dectris Pilatus3 R 200K) and employing confocal multilayer optic-monochromated Mo-Ka radiation $(\lambda=0.71073 \AA)$ at a temperature of 100K. Rotation frames were integrated using CrysAlisPro, ${ }^{1}$ producing a listing of unaveraged $\mathrm{F}^{2}$ and $\sigma\left(F^{2}\right)$ values. The intensity data were corrected for Lorentz and polarization effects and for absorption using SCALE3 ABSPACK ${ }^{2}$ (minimum and maximum transmission 0.7186, 1.0000). The structure was solved by dual space methods - SheIXT. ${ }^{3}$ Refinement was by full-matrix least squares based on $\mathrm{F} 2$ using SHELXL-2018. ${ }^{3} \mathrm{All}$ reflections were used during refinement. Nonhydrogen atoms were refined anisotropically and hydrogen atoms were refined using a riding model. Structures were rendered using Mercury version 2021.1 at 50\% probability ellipsoids.

Table S2. XRD collection parameters.

\begin{tabular}{|c|c|c|}
\hline & $1-\mathrm{Nb}$ & 1-Ta \\
\hline Empirical formula & $\mathrm{C}_{70} \mathrm{H}_{55} \mathrm{~N}_{3} \mathrm{NaNbO}_{7}$ & $\mathrm{C}_{70} \mathrm{H}_{55} \mathrm{~N}_{3} \mathrm{NaO}_{7} \mathrm{Ta}$ \\
\hline Formula weight & 1166.07 & 1254.11 \\
\hline Temperature/K & 100 & 100 \\
\hline Crystal system & trigonal & trigonal \\
\hline Space group & R3 & R3 \\
\hline a & $15.5338(4) \dot{A}$ & $15.5173(2) \dot{A}$ \\
\hline c & $22.0012(7) \AA$ & $22.0769(3) \AA$ \\
\hline Volume & $4597.6(3) \AA^{3}$ & $4603.64(13) \AA^{3}$ \\
\hline Z & 3 & 3 \\
\hline$d_{\text {calc }}$ & $1.263 \mathrm{~g} / \mathrm{cm}^{3}$ & $1.357 \mathrm{~g} / \mathrm{cm}^{3}$ \\
\hline$F(000)$ & 1812.0 & 1908.0 \\
\hline Crystal size, mm & $0.31 \times 0.13 \times 0.1$ & $0.25 \times 0.2 \times 0.2$ \\
\hline $2 \theta$ range for data collection & $4.784-56.548^{\circ}$ & $4.776-56.562^{\circ}$ \\
\hline Reflections collected & 31115 & 47088 \\
\hline Goodness-of-fit on $F^{2}$ & 1.088 & 1.078 \\
\hline Final $R$ indexes $[l>=2 \sigma(I)]$ & $\mathrm{R}_{1}=0.0379, \mathrm{wR} 2=0.1012$ & $\mathrm{R}_{1}=0.0199, \mathrm{wR}_{2}=0.0518$ \\
\hline
\end{tabular}




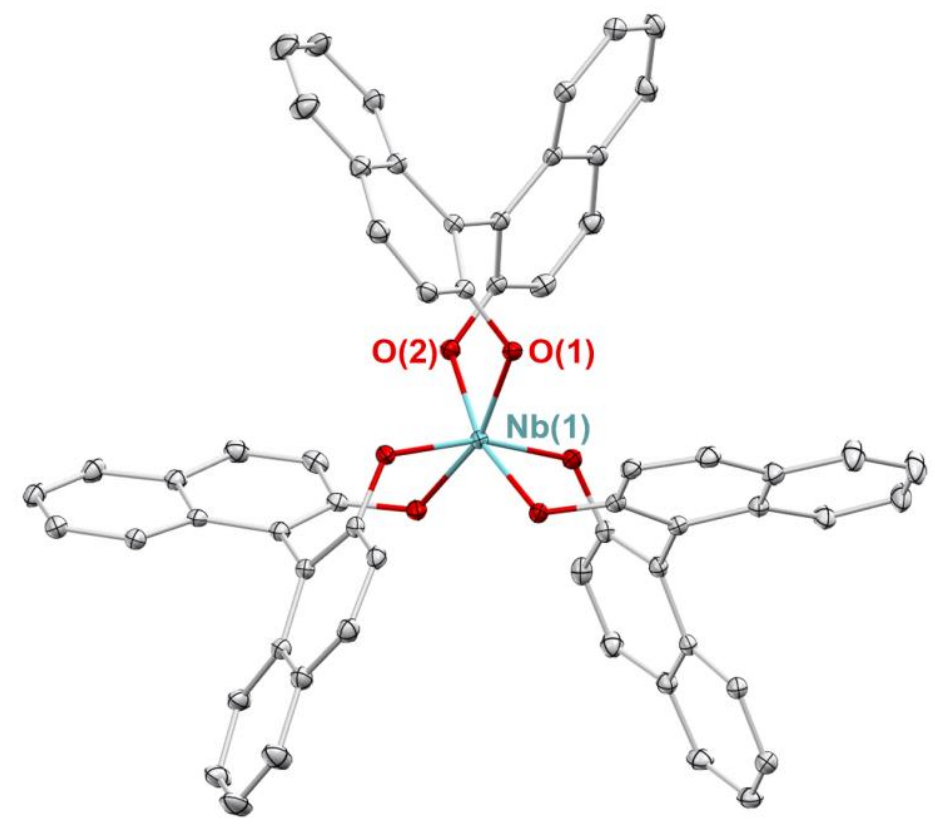

Figure S12. Solid state crystal structure of $\mathbf{1 - N b}$. Thermal ellipsoids are depicted at $50 \%$ probability and counterions and hydrogen atoms are omitted for clarity.

Table S3. Selected bond angles in 1-Nb.

\begin{tabular}{llllll}
\hline O1-Nb1-O11 $^{-87.80(12)}$ & O1-Nb1-O1 $^{1}$ & $87.80(12)$ & O1-Nb1-O1 $^{2}$ & $87.80(12)$ \\
\hline O2-Nb1-O11 $^{163.10(9)}$ & O2-Nb1-O1 $^{2}$ & $81.91(10)$ & O2-Nb1-O1 $^{2}$ & $163.10(9)$ \\
\hline O2-Nb1-O1 & $105.07(10)$ & O2-Nb1-O1 & $163.10(9)$ & O2-Nb1-O11 $^{105.07(10)}$ \\
\hline O2-Nb1-O1 & $81.91(10)$ & O2-Nb1-O11 $^{1}$ & $81.91(10)$ & O2-Nb1-O12 $^{2}$ & $105.07(10)$ \\
\hline O2-Nb1-O2 & $87.84(11)$ & O2-Nb1-O2 & $87.84(11)$ & O2-Nb1-O2 $^{2}$ & $87.84(11)$ \\
\hline C1-O1-Nb1 & $125.5(2)$ & & & &
\end{tabular}

Table S4. Selected bond distances in 1-Nb.

\begin{tabular}{llllll} 
Nb1-01 & $1.977(3)$ & Nb1-01 & $1.977(3)$ & Nb1-01 & $1.977(3)$ \\
\hline Nb1-02 & $1.976(3)$ & Nb1-02 & $1.976(3)$ & Nb1-02 & $1.976(3)$
\end{tabular}




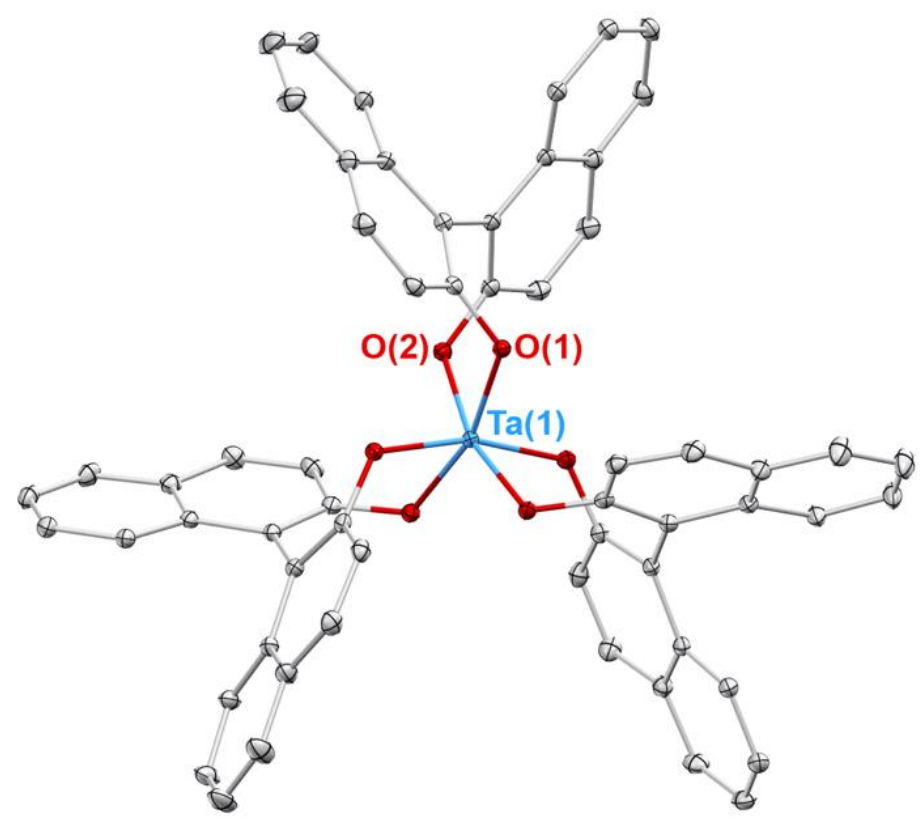

Figure S13. Solid state crystal structure of 1-Ta. Thermal ellipsoids are depicted at 50\% probability and counterions and hydrogen atoms are omitted for clarity.

Table S5. Selected bond angles in 1-Ta.

\begin{tabular}{llllll}
\hline O1-Ta1-O1 $^{1}$ & $87.95(12)$ & O1-Ta1-O1 $^{1}$ & $87.94(12)$ & O1-Ta1-O1 $^{2}$ & $87.94(12)$ \\
\hline O1-Ta1-O2 $^{2}$ & $82.35(9)$ & O1-Ta1-O2 $^{1}$ & $104.45(9)$ & O1-Ta1-O2 $^{2}$ & $104.46(9)$ \\
\hline O1-Ta1-O2 $^{1}$ & $82.35(9)$ & O1-Ta1-O2 $^{1}$ & $163.88(8)$ & O1-Ta1-O2 $^{163.88(8)}$ \\
\hline O1-Ta1-O2 $^{-82.35(9)}$ & O1-Ta1-O2 $^{1}$ & $104.46(9)$ & O1-Ta1-O2 $^{2}$ & $163.88(8)$ \\
\hline O2-Ta1-O2 $^{1}$ & $87.63(12)$ & O2-Ta1-O2 $^{1}$ & $87.63(12)$ & O2-Ta1-O2 $^{2}$ & $87.63(12)$ \\
\hline
\end{tabular}

Table S6. Selected bond distances in 1-Ta.

\begin{tabular}{llllll}
\hline Ta1-01 & $1.974(3)$ & Ta1-01 & $1.974(3)$ & Ta1-01 & $1.974(3)$ \\
\hline Ta1-02 & $1.975(3)$ & Ta1-02 & $1.976(3)$ & Ta1-02 & $1.976(3)$ \\
\hline
\end{tabular}




\section{DFT Calculations}

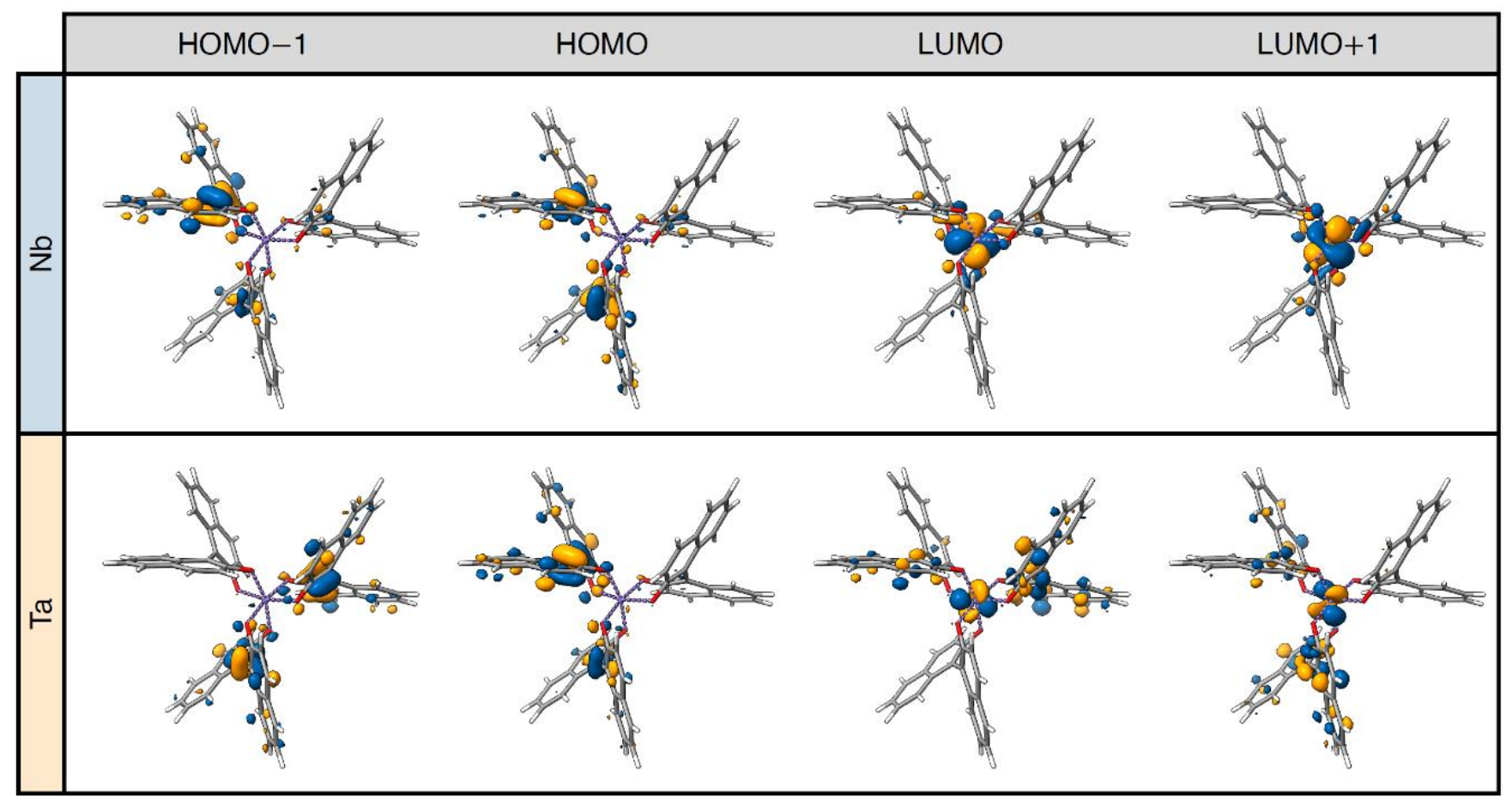

Figure S14. Selected calculated molecular orbitals for $\mathbf{1 - M}$ plotted at an isovalue of 0.04 . The HOMO/HOMO-1 orbitals are ligand-based for both 1-Nb and 1-Ta, while the LUMO/LUMO+1 contain substantial d-orbital character for both complexes. 
Table S7. XYZ coordinates and Gibbs free energy for the DFT structure of 1-Nb.

$E_{h}=-2813.2628174323$

\begin{tabular}{cccc}
\hline Atom & $\mathbf{X}$ & $\mathbf{Y}$ & $\mathbf{Z}$ \\
\hline $\mathbf{N b}$ & 7.99999 & 13.40887 & 11.02175 \\
\hline $\mathbf{O}$ & 7.050633 & 12.14312 & 12.23 \\
\hline $\mathbf{O}$ & 8.226172 & 11.74229 & 9.965327 \\
\hline $\mathbf{C}$ & 7.349325 & 10.90064 & 12.64425 \\
\hline $\mathbf{C}$ & 7.785597 & 10.75453 & 13.99418 \\
\hline $\mathbf{H}$ & 7.838827 & 11.65517 & 14.60926 \\
\hline $\mathbf{C}$ & 8.12369 & 9.522654 & 14.49683 \\
\hline $\mathbf{H}$ & 8.448772 & 9.417994 & 15.5357 \\
\hline $\mathbf{C}$ & 8.092148 & 8.368183 & 13.66517 \\
\hline $\mathbf{C}$ & 8.486313 & 7.094444 & 14.16306 \\
\hline $\mathbf{H}$ & 8.791478 & 7.019064 & 15.2109 \\
\hline $\mathbf{C}$ & 8.507811 & 5.982648 & 13.34628 \\
\hline $\mathbf{H}$ & 8.819986 & 5.01256 & 13.74235 \\
\hline $\mathbf{C}$ & 8.151704 & 6.117297 & 11.98077 \\
\hline $\mathbf{H}$ & 8.199222 & 5.245918 & 11.32162 \\
\hline $\mathbf{C}$ & 7.751567 & 7.33857 & 11.47199 \\
\hline $\mathbf{H}$ & 7.489961 & 7.417372 & 10.41615 \\
\hline $\mathbf{C}$ & 7.67841 & 8.504617 & 12.29361 \\
\hline $\mathbf{C}$ & 7.239058 & 9.787258 & 11.79744 \\
\hline $\mathbf{C}$ & 6.683261 & 9.96008 & 10.41911 \\
\hline $\mathbf{C}$ & 5.530169 & 9.220554 & 9.95826 \\
\hline $\mathbf{C}$ & 4.800609 & 8.318174 & 10.79142 \\
\hline $\mathbf{H}$ & 5.120042 & 8.166924 & 11.82249 \\
\hline $\mathbf{C}$ & 3.694636 & 7.633533 & 10.32444 \\
\hline $\mathbf{H}$ & 3.160467 & 6.952898 & 10.99335 \\
\hline $\mathbf{C}$ & 3.238723 & 7.813376 & 8.994802 \\
\hline $\mathbf{H}$ & 2.362562 & 7.266785 & 8.635868 \\
\hline $\mathbf{C}$ & 3.900408 & 8.69705 & 8.166939 \\
\hline $\mathbf{H}$ & 3.547466 & 8.873958 & 7.146629 \\
\hline & & & \\
\hline & & \\
\hline $\mathbf{H}$ & & \\
\hline $\mathbf{H}$ & & \\
\hline
\end{tabular}




\begin{tabular}{cccc}
\hline $\mathbf{C}$ & 5.042847 & 9.416625 & 8.617719 \\
\hline $\mathbf{C}$ & 5.700433 & 10.34907 & 7.766211 \\
\hline $\mathbf{H}$ & 5.327612 & 10.4955 & 6.74836 \\
\hline $\mathbf{C}$ & 6.767586 & 11.07904 & 8.225875 \\
\hline $\mathbf{H}$ & 7.267435 & 11.8184 & 7.597491 \\
\hline $\mathbf{C}$ & 7.24242 & 10.91223 & 9.558059 \\
\hline $\mathbf{O}$ & 9.652654 & 13.32618 & 12.119 \\
\hline $\mathbf{O}$ & 9.205227 & 14.46721 & 9.840318 \\
\hline $\mathbf{C}$ & 10.41418 & 14.36062 & 12.53098 \\
\hline $\mathbf{C}$ & 10.2011 & 14.82883 & 13.85931 \\
\hline $\mathbf{H}$ & 9.481781 & 14.28957 & 14.4784 \\
\hline $\mathbf{C}$ & 10.8677 & 15.93221 & 14.32935 \\
\hline $\mathbf{H}$ & 10.68744 & 16.29582 & 15.345 \\
\hline $\mathbf{C}$ & 11.77845 & 16.6367 & 13.49182 \\
\hline $\mathbf{C}$ & 12.43826 & 17.80945 & 13.95604 \\
\hline $\mathbf{H}$ & 12.23015 & 18.15059 & 14.9745 \\
\hline $\mathbf{C}$ & 13.30319 & 18.51294 & 13.14287 \\
\hline $\mathbf{H}$ & 13.80664 & 19.41005 & 13.51275 \\
\hline $\mathbf{C}$ & 13.51944 & 18.07169 & 11.81378 \\
\hline $\mathbf{H}$ & 14.18336 & 18.63956 & 11.15581 \\
\hline $\mathbf{C}$ & 12.89079 & 16.93861 & 11.33316 \\
\hline $\mathbf{H}$ & 13.06824 & 16.63234 & 10.30241 \\
\hline $\mathbf{C}$ & 12.01563 & 16.16263 & 12.15321 \\
\hline $\mathbf{C}$ & 11.34398 & 14.97245 & 11.68179 \\
\hline $\mathbf{C}$ & 11.56477 & 14.43301 & 10.30399 \\
\hline $\mathbf{C}$ & 12.87847 & 14.07739 & 9.821451 \\
\hline $\mathbf{C}$ & 14.03119 & 14.0479 & 10.66382 \\
\hline $\mathbf{H}$ & 13.91839 & 14.27594 & 11.72456 \\
\hline $\mathbf{C}$ & 15.28238 & 13.73041 & 10.16937 \\
\hline $\mathbf{C}$ & 16.14283 & 13.71359 & 10.8441 \\
\hline & 15.46216 & 13.42014 & 8.798092 \\
\hline $\mathbf{H}$ & 16.45577 & 13.17498 & 8.413182 \\
\hline $\mathbf{H}$ & & & \\
\hline $\mathbf{C}$ & 146628 & 13.40058 & 7.960115 \\
\hline $\mathbf{C}$ & & \\
\hline $\mathbf{H}$ & & \\
\hline
\end{tabular}




\begin{tabular}{|c|c|c|c|}
\hline H & 14.47863 & 13.129 & 6.906231 \\
\hline C & 13.06265 & 13.7062 & 8.443427 \\
\hline C & 11.92636 & 13.63008 & 7.590339 \\
\hline H & 12.06693 & 13.34161 & 6.544767 \\
\hline C & 10.66777 & 13.87703 & 8.080784 \\
\hline $\mathbf{H}$ & 9.782844 & 13.78344 & 7.447734 \\
\hline C & 10.47287 & 14.26716 & 9.438869 \\
\hline 0 & 7.258798 & 14.8956 & 12.11857 \\
\hline 0 & 6.496003 & 13.92451 & 9.846352 \\
\hline C & 5.987852 & 15.03734 & 12.54496 \\
\hline C & 5.714185 & 14.71039 & 13.90483 \\
\hline $\mathbf{H}$ & 6.556608 & 14.43054 & 14.54065 \\
\hline C & 4.429755 & 14.73578 & 14.39108 \\
\hline $\mathbf{H}$ & 4.227395 & 14.48337 & 15.43595 \\
\hline C & 3.338941 & 15.04096 & 13.52994 \\
\hline C & 1.994485 & 14.98228 & 13.99518 \\
\hline H & 1.817004 & 14.72556 & 15.04381 \\
\hline C & 0.934938 & 15.21281 & 13.14242 \\
\hline H & -0.0929 & 15.15455 & 13.51143 \\
\hline C & 1.187328 & 15.50685 & 11.77919 \\
\hline $\mathbf{H}$ & 0.348683 & 15.66433 & 11.09565 \\
\hline C & 2.481758 & 15.59436 & 11.30382 \\
\hline $\mathbf{H}$ & 2.652428 & 15.8125 & 10.24846 \\
\hline C & 3.605338 & 15.38621 & 12.15858 \\
\hline C & 4.965225 & 15.45937 & 11.68309 \\
\hline C & 5.307299 & 15.94579 & 10.30916 \\
\hline C & 4.933031 & 17.26146 & 9.845892 \\
\hline C & 4.229728 & 18.20125 & 10.6595 \\
\hline H & 3.971639 & 17.93251 & 11.68373 \\
\hline C & 3.866131 & 19.44446 & 10.17813 \\
\hline H & 3.324534 & 20.13781 & 10.82794 \\
\hline C & 4.195238 & 19.8355 & 8.856132 \\
\hline $\mathbf{H}$ & 3.893793 & 20.81823 & 8.484079 \\
\hline
\end{tabular}




\begin{tabular}{cccc}
\hline $\mathbf{C}$ & 4.908747 & 18.97098 & 8.051873 \\
\hline $\mathbf{H}$ & 5.200498 & 19.26609 & 7.039609 \\
\hline $\mathbf{C}$ & 5.295113 & 17.6818 & 8.516703 \\
\hline $\mathbf{C}$ & 6.048743 & 16.80261 & 7.688059 \\
\hline $\mathbf{H}$ & 6.333927 & 17.13097 & 6.684819 \\
\hline $\mathbf{C}$ & 6.428335 & 15.56607 & 8.147393 \\
\hline $\mathbf{H}$ & 7.018198 & 14.88325 & 7.532387 \\
\hline $\mathbf{C}$ & 6.066056 & 15.13521 & 9.456346 \\
\hline
\end{tabular}


Table S8. XYZ coordinates and Gibbs free energy for the DFT structure of [1-Nb]$E_{h}=-2813.35176186857$

\begin{tabular}{cccc}
\hline Atom & $\mathbf{X}$ & $\mathbf{Y}$ & $\mathbf{Z}$ \\
\hline $\mathbf{N b}$ & 8.049647 & 13.40591 & 11.06471 \\
\hline $\mathbf{O}$ & 7.087777 & 12.17286 & 12.34203 \\
\hline $\mathbf{O}$ & 8.548218 & 11.60767 & 10.11008 \\
\hline $\mathbf{C}$ & 7.315144 & 10.92682 & 12.7539 \\
\hline $\mathbf{C}$ & 7.639421 & 10.74169 & 14.13424 \\
\hline $\mathbf{H}$ & 7.679575 & 11.63546 & 14.7614 \\
\hline $\mathbf{C}$ & 7.883006 & 9.493174 & 14.6521 \\
\hline $\mathbf{H}$ & 8.120572 & 9.368233 & 15.71292 \\
\hline $\mathbf{C}$ & 7.859593 & 8.344936 & 13.81191 \\
\hline $\mathbf{C}$ & 8.138018 & 7.047197 & 14.32644 \\
\hline $\mathbf{H}$ & 8.355583 & 6.948268 & 15.39462 \\
\hline $\mathbf{C}$ & 8.15385 & 5.938116 & 13.505 \\
\hline $\mathbf{H}$ & 8.373494 & 4.948797 & 13.91593 \\
\hline $\mathbf{C}$ & 7.910891 & 6.099762 & 12.11748 \\
\hline $\mathbf{H}$ & 7.953453 & 5.229229 & 11.45653 \\
\hline $\mathbf{C}$ & 7.628533 & 7.346251 & 11.59068 \\
\hline $\mathbf{H}$ & 7.453313 & 7.447351 & 10.51855 \\
\hline $\mathbf{C}$ & 7.561328 & 8.513415 & 12.4131 \\
\hline $\mathbf{C}$ & 7.229294 & 9.816466 & 11.89196 \\
\hline $\mathbf{C}$ & 6.799685 & 10.01027 & 10.47092 \\
\hline $\mathbf{C}$ & 5.621534 & 9.368333 & 9.940093 \\
\hline $\mathbf{C}$ & 4.761608 & 8.541943 & 10.72885 \\
\hline $\mathbf{H}$ & 5.001397 & 8.377077 & 11.77979 \\
\hline $\mathbf{C}$ & 3.633604 & 7.946209 & 10.19506 \\
\hline $\mathbf{H}$ & 3.000448 & 7.321626 & 10.83241 \\
\hline $\mathbf{C}$ & 3.279469 & 8.147818 & 8.837743 \\
\hline $\mathbf{H}$ & 2.383021 & 7.675761 & 8.426481 \\
\hline $\mathbf{C}$ & 4.071667 & 8.958803 & 8.048361 \\
\hline $\mathbf{H}$ & 3.800126 & 9.154407 & 7.005969 \\
\hline & & & \\
\hline
\end{tabular}




\begin{tabular}{cccc}
\hline $\mathbf{C}$ & 5.243877 & 9.577981 & 8.565507 \\
\hline $\mathbf{C}$ & 6.053002 & 10.42119 & 7.749907 \\
\hline $\mathbf{H}$ & 5.767082 & 10.58052 & 6.705392 \\
\hline $\mathbf{C}$ & 7.152824 & 11.05123 & 8.273143 \\
\hline $\mathbf{H}$ & 7.765747 & 11.7217 & 7.66611 \\
\hline $\mathbf{C}$ & 7.526353 & 10.88967 & 9.64565 \\
\hline $\mathbf{O}$ & 9.866524 & 13.1432 & 12.08194 \\
\hline $\mathbf{O}$ & 9.235845 & 14.53369 & 9.840237 \\
\hline $\mathbf{C}$ & 10.54143 & 14.20162 & 12.51375 \\
\hline $\mathbf{C}$ & 10.36023 & 14.61461 & 13.87291 \\
\hline $\mathbf{H}$ & 9.693529 & 14.00903 & 14.49164 \\
\hline $\mathbf{C}$ & 10.97791 & 15.73288 & 14.3726 \\
\hline $\mathbf{H}$ & 10.80966 & 16.04315 & 15.40863 \\
\hline $\mathbf{C}$ & 11.82595 & 16.52309 & 13.54406 \\
\hline $\mathbf{C}$ & 12.44667 & 17.70292 & 14.0413 \\
\hline $\mathbf{H}$ & 12.25012 & 17.9924 & 15.07861 \\
\hline $\mathbf{C}$ & 13.2622 & 18.47826 & 13.24031 \\
\hline $\mathbf{H}$ & 13.73742 & 19.3794 & 13.63728 \\
\hline $\mathbf{C}$ & 13.46336 & 18.1007 & 11.88905 \\
\hline $\mathbf{H}$ & 14.08975 & 18.72135 & 11.24128 \\
\hline $\mathbf{C}$ & 12.86623 & 16.96501 & 11.37425 \\
\hline $\mathbf{H}$ & 13.03107 & 16.70724 & 10.32772 \\
\hline $\mathbf{C}$ & 12.04071 & 16.11747 & 12.17772 \\
\hline $\mathbf{C}$ & 11.41039 & 14.92329 & 11.67091 \\
\hline $\mathbf{C}$ & 11.60143 & 14.47058 & 10.25652 \\
\hline $\mathbf{C}$ & 12.90381 & 14.15811 & 9.722413 \\
\hline $\mathbf{C}$ & 14.08109 & 14.10937 & 10.5318 \\
\hline $\mathbf{H}$ & 13.98882 & 14.28621 & 11.6046 \\
\hline $\mathbf{C}$ & 15.32549 & 13.8404 & 9.992692 \\
\hline $\mathbf{H}$ & 16.20338 & 13.80999 & 10.64455 \\
\hline $\mathbf{H}$ & 15.47593 & 13.59513 & 8.604273 \\
\hline & 16.46339 & 13.38629 & 8.183372 \\
\hline $\mathbf{C}$ & 14.35775 & 13.59453 & 7.794975 \\
\hline & & & \\
\hline $\mathbf{C}$ & & \\
\hline $\mathbf{C}$ & & \\
\hline $\mathbf{H}$ & & \\
\hline
\end{tabular}




\begin{tabular}{|c|c|c|c|}
\hline H & 14.44713 & 13.3744 & 6.7264 \\
\hline C & 13.06226 & 13.85627 & 8.322731 \\
\hline C & 11.9057 & 13.81128 & 7.495052 \\
\hline $\mathbf{H}$ & 12.02327 & 13.57533 & 6.43292 \\
\hline C & 10.65885 & 14.0272 & 8.028133 \\
\hline $\mathbf{H}$ & 9.759547 & 13.9637 & 7.410927 \\
\hline C & 10.48013 & 14.34756 & 9.411469 \\
\hline 0 & 7.350215 & 15.03519 & 12.15354 \\
\hline 0 & 6.506893 & 13.82884 & 9.806243 \\
\hline C & 6.08333 & 15.11689 & 12.55686 \\
\hline C & 5.821917 & 14.88222 & 13.9454 \\
\hline $\mathbf{H}$ & 6.687343 & 14.70756 & 14.58955 \\
\hline C & 4.544834 & 14.86315 & 14.44735 \\
\hline $\mathbf{H}$ & 4.367606 & 14.68253 & 15.51232 \\
\hline C & 3.428559 & 15.0357 & 13.58139 \\
\hline C & 2.09462 & 14.93946 & 14.0673 \\
\hline H & 1.942979 & 14.74983 & 15.13483 \\
\hline C & 1.012445 & 15.05644 & 13.2182 \\
\hline H & -0.00666 & 14.97118 & 13.60591 \\
\hline C & 1.236897 & 15.26948 & 11.83501 \\
\hline $\mathbf{H}$ & 0.384027 & 15.3383 & 11.15369 \\
\hline C & 2.521275 & 15.38828 & 11.33726 \\
\hline H & 2.669413 & 15.54459 & 10.26702 \\
\hline C & 3.667916 & 15.2957 & 12.18489 \\
\hline C & 5.015089 & 15.41616 & 11.68583 \\
\hline C & 5.304082 & 15.85002 & 10.28155 \\
\hline C & 4.872587 & 17.13552 & 9.787001 \\
\hline C & 4.146369 & 18.07246 & 10.58666 \\
\hline H & 3.919885 & 17.82207 & 11.62306 \\
\hline C & 3.717155 & 19.28381 & 10.07789 \\
\hline H & 3.158621 & 19.97156 & 10.71993 \\
\hline C & 4.000609 & 19.65074 & 8.738438 \\
\hline $\mathbf{H}$ & 3.650292 & 20.6088 & 8.344992 \\
\hline
\end{tabular}




\begin{tabular}{cccc}
\hline $\mathbf{C}$ & 4.733959 & 18.79133 & 7.944977 \\
\hline $\mathbf{H}$ & 4.992378 & 19.068 & 6.918017 \\
\hline $\mathbf{C}$ & 5.183336 & 17.53363 & 8.436708 \\
\hline $\mathbf{C}$ & 5.946738 & 16.65556 & 7.615118 \\
\hline $\mathbf{H}$ & 6.197805 & 16.96468 & 6.596111 \\
\hline $\mathbf{C}$ & 6.370303 & 15.44381 & 8.098859 \\
\hline $\mathbf{H}$ & 6.95902 & 14.75682 & 7.4862 \\
\hline $\mathbf{C}$ & 6.059737 & 15.02242 & 9.430647 \\
\hline
\end{tabular}


Table S9. XYZ coordinates and Gibbs free energy for the DFT structure of [1-Nb]+. $E_{h}=-2813.076351080123$

\begin{tabular}{cccc}
\hline Atom & $\mathbf{X}$ & $\mathbf{Y}$ & $\mathbf{Z}$ \\
\hline $\mathbf{N b}$ & 8.13672 & 13.5049 & 11.0012 \\
\hline $\mathbf{O}$ & 7.15213 & 12.2199 & 12.2812 \\
\hline $\mathbf{O}$ & 8.31382 & 11.7729 & 10.0043 \\
\hline $\mathbf{C}$ & 7.31914 & 10.9956 & 12.6869 \\
\hline $\mathbf{C}$ & 7.54531 & 10.7841 & 14.0818 \\
\hline $\mathbf{H}$ & 7.57601 & 11.6674 & 14.7217 \\
\hline $\mathbf{C}$ & 7.71045 & 9.52387 & 14.5906 \\
\hline $\mathbf{H}$ & 7.88228 & 9.38194 & 15.6611 \\
\hline $\mathbf{C}$ & 7.70795 & 8.37122 & 13.7392 \\
\hline $\mathbf{C}$ & 7.95886 & 7.08533 & 14.2636 \\
\hline $\mathbf{H}$ & 8.11906 & 6.97634 & 15.3396 \\
\hline $\mathbf{C}$ & 8.02513 & 5.97514 & 13.4279 \\
\hline $\mathbf{H}$ & 8.22198 & 4.98579 & 13.8481 \\
\hline $\mathbf{C}$ & 7.87483 & 6.14125 & 12.0388 \\
\hline $\mathbf{H}$ & 7.96942 & 5.27933 & 11.374 \\
\hline $\mathbf{C}$ & 7.61919 & 7.39747 & 11.5024 \\
\hline $\mathbf{H}$ & 7.53158 & 7.5074 & 10.4207 \\
\hline $\mathbf{C}$ & 7.48367 & 8.54119 & 12.3341 \\
\hline $\mathbf{C}$ & 7.19442 & 9.85602 & 11.8005 \\
\hline $\mathbf{C}$ & 6.69604 & 10.0456 & 10.4365 \\
\hline $\mathbf{C}$ & 5.55983 & 9.29832 & 9.9158 \\
\hline $\mathbf{C}$ & 4.74249 & 8.46238 & 10.7246 \\
\hline $\mathbf{H}$ & 4.97167 & 8.33777 & 11.7827 \\
\hline $\mathbf{C}$ & 3.64262 & 7.79943 & 10.2007 \\
\hline $\mathbf{H}$ & 3.03995 & 7.16316 & 10.8539 \\
\hline $\mathbf{C}$ & 3.28523 & 7.95106 & 8.84456 \\
\hline $\mathbf{H}$ & 2.41513 & 7.42693 & 8.44179 \\
\hline $\mathbf{C}$ & 4.03522 & 8.7892 & 8.03657 \\
\hline $\mathbf{H}$ & 3.75259 & 8.95628 & 6.99365 \\
\hline & & & \\
\hline $\mathbf{H}$ & & & \\
\hline $\mathbf{H}$ & & \\
\hline $\mathbf{H}$ & & \\
\hline
\end{tabular}




\begin{tabular}{cccc}
\hline $\mathbf{C}$ & 5.16839 & 9.47189 & 8.54648 \\
\hline $\mathbf{C}$ & 5.90687 & 10.3653 & 7.71334 \\
\hline $\mathbf{H}$ & 5.6048 & 10.4823 & 6.66892 \\
\hline $\mathbf{C}$ & 6.95571 & 11.0972 & 8.21109 \\
\hline $\mathbf{H}$ & 7.51627 & 11.7921 & 7.58439 \\
\hline $\mathbf{C}$ & 7.33083 & 10.9907 & 9.57626 \\
\hline $\mathbf{O}$ & 9.76852 & 13.386 & 12.0951 \\
\hline $\mathbf{O}$ & 9.31514 & 14.5417 & 9.83038 \\
\hline $\mathbf{C}$ & 10.5615 & 14.3933 & 12.528 \\
\hline $\mathbf{C}$ & 10.3772 & 14.8188 & 13.8732 \\
\hline $\mathbf{H}$ & 9.66867 & 14.2647 & 14.4921 \\
\hline $\mathbf{C}$ & 11.0595 & 15.9053 & 14.3596 \\
\hline $\mathbf{H}$ & 10.9018 & 16.2414 & 15.3882 \\
\hline $\mathbf{C}$ & 11.9566 & 16.628 & 13.5231 \\
\hline $\mathbf{C}$ & 12.6286 & 17.7859 & 14.0064 \\
\hline $\mathbf{H}$ & 12.4383 & 18.1019 & 15.0363 \\
\hline $\mathbf{C}$ & 13.4826 & 18.5063 & 13.1968 \\
\hline $\mathbf{H}$ & 13.9954 & 19.392 & 13.5808 \\
\hline $\mathbf{C}$ & 13.6761 & 18.0977 & 11.854 \\
\hline $\mathbf{H}$ & 14.3323 & 18.6791 & 11.2004 \\
\hline $\mathbf{C}$ & 13.0349 & 16.98 & 11.3545 \\
\hline $\mathbf{H}$ & 13.1948 & 16.6995 & 10.3136 \\
\hline $\mathbf{C}$ & 12.1684 & 16.1891 & 12.1686 \\
\hline $\mathbf{C}$ & 11.4795 & 15.0164 & 11.6771 \\
\hline $\mathbf{C}$ & 11.6822 & 14.5074 & 10.2845 \\
\hline $\mathbf{C}$ & 12.9928 & 14.1594 & 9.78478 \\
\hline $\mathbf{C}$ & 14.1482 & 14.1031 & 10.6212 \\
\hline $\mathbf{H}$ & 14.0415 & 14.3027 & 11.6882 \\
\hline $\mathbf{C}$ & 15.3953 & 13.7928 & 10.1123 \\
\hline $\mathbf{H}$ & 16.2588 & 13.7535 & 10.782 \\
\hline $\mathbf{C}$ & 15.5665 & 13.5169 & 8.7328 \\
\hline $\mathbf{H}$ & 16.5571 & 13.2769 & 8.33722 \\
\hline & 14.4672 & 13.5232 & 7.89951 \\
\hline & & & \\
\hline $\mathbf{C}$ & & & \\
\hline & & & \\
\hline
\end{tabular}




\begin{tabular}{cccc}
\hline H & 14.5732 & 13.2776 & 6.83873 \\
\hline $\mathbf{C}$ & 13.1676 & 13.8229 & 8.39717 \\
\hline $\mathbf{C}$ & 12.0279 & 13.7738 & 7.54693 \\
\hline $\mathbf{H}$ & 12.1624 & 13.5136 & 6.49336 \\
\hline $\mathbf{C}$ & 10.7725 & 14.0107 & 8.04989 \\
\hline $\mathbf{H}$ & 9.8839 & 13.9382 & 7.41934 \\
\hline $\mathbf{C}$ & 10.5911 & 14.3615 & 9.41813 \\
\hline $\mathbf{O}$ & 7.36725 & 14.9558 & 12.0726 \\
\hline $\mathbf{O}$ & 6.6277 & 13.9253 & 9.82355 \\
\hline $\mathbf{C}$ & 6.10046 & 15.0784 & 12.538 \\
\hline $\mathbf{C}$ & 5.90128 & 14.8387 & 13.9263 \\
\hline $\mathbf{H}$ & 6.78239 & 14.6518 & 14.5436 \\
\hline $\mathbf{C}$ & 4.63751 & 14.8383 & 14.4631 \\
\hline $\mathbf{H}$ & 4.48875 & 14.6603 & 15.5319 \\
\hline $\mathbf{C}$ & 3.50132 & 15.0169 & 13.6259 \\
\hline $\mathbf{C}$ & 2.18132 & 14.9193 & 14.1501 \\
\hline $\mathbf{H}$ & 2.05955 & 14.733 & 15.2214 \\
\hline $\mathbf{C}$ & 1.07859 & 15.0274 & 13.3286 \\
\hline $\mathbf{H}$ & 0.07091 & 14.9396 & 13.7439 \\
\hline $\mathbf{C}$ & 1.26179 & 15.2305 & 11.9382 \\
\hline $\mathbf{H}$ & 0.39003 & 15.286 & 11.2807 \\
\hline $\mathbf{C}$ & 2.53021 & 15.3546 & 11.4041 \\
\hline $\mathbf{H}$ & 2.64667 & 15.5011 & 10.3291 \\
\hline $\mathbf{C}$ & 3.69563 & 15.2758 & 12.224 \\
\hline $\mathbf{C}$ & 5.03211 & 15.398 & 11.6896 \\
\hline $\mathbf{C}$ & 5.28799 & 15.8536 & 10.2856 \\
\hline $\mathbf{C}$ & 4.78825 & 17.1184 & 9.79427 \\
\hline $\mathbf{C}$ & 4.052 & 18.0327 & 10.6073 \\
\hline $\mathbf{H}$ & 3.8662 & 17.7888 & 11.6529 \\
\hline $\mathbf{C}$ & 3.56386 & 19.221 & 10.0987 \\
\hline $\mathbf{H}$ & 2.99942 & 19.8947 & 10.7496 \\
\hline $\mathbf{C}$ & 3.79716 & 19.5836 & 8.74899 \\
\hline & 3.40182 & 20.5244 & 8.35725 \\
\hline $\mathbf{H}$ & & & \\
\hline $\mathbf{H}$ & & & \\
\hline
\end{tabular}




\begin{tabular}{cccc}
\hline $\mathbf{C}$ & 4.54269 & 18.7481 & 7.94316 \\
\hline $\mathbf{H}$ & 4.76684 & 19.0261 & 6.90908 \\
\hline $\mathbf{C}$ & 5.05282 & 17.5135 & 8.43483 \\
\hline $\mathbf{C}$ & 5.83392 & 16.6664 & 7.59859 \\
\hline $\mathbf{H}$ & 6.04442 & 16.9778 & 6.57193 \\
\hline $\mathbf{C}$ & 6.33274 & 15.483 & 8.0803 \\
\hline $\mathbf{H}$ & 6.94334 & 14.823 & 7.46036 \\
\hline $\mathbf{C}$ & 6.07012 & 15.0802 & 9.42025 \\
\hline
\end{tabular}


Table S10. XYZ coordinates and Gibbs free energy for the DFT structure of 1-Ta. $E_{h}=-2813.329820426888$

\begin{tabular}{cccc}
\hline Atom & $\mathbf{X}$ & $\mathbf{Y}$ & $\mathbf{Z}$ \\
\hline $\mathbf{T a}$ & 0.22181 & 8.92192 & 10.8784 \\
\hline $\mathbf{O}$ & 1.88192 & 8.81369 & 11.9818 \\
\hline $\mathbf{O}$ & 1.4337 & 9.99651 & 9.70645 \\
\hline $\mathbf{C}$ & 2.65139 & 9.83776 & 12.4089 \\
\hline $\mathbf{C}$ & 2.45035 & 10.2801 & 13.7476 \\
\hline $\mathbf{H}$ & 1.73642 & 9.72913 & 14.3628 \\
\hline $\mathbf{C}$ & 3.11999 & 11.3746 & 14.2336 \\
\hline $\mathbf{H}$ & 2.94652 & 11.7195 & 15.257 \\
\hline $\mathbf{C}$ & 4.02476 & 12.0945 & 13.4029 \\
\hline $\mathbf{C}$ & 4.68731 & 13.2589 & 13.8838 \\
\hline $\mathbf{H}$ & 4.48386 & 13.583 & 14.9089 \\
\hline $\mathbf{C}$ & 5.54895 & 13.9756 & 13.0787 \\
\hline $\mathbf{H}$ & 6.05477 & 14.866 & 13.4613 \\
\hline $\mathbf{C}$ & 5.75913 & 13.5559 & 11.7417 \\
\hline $\mathbf{H}$ & 6.4214 & 14.1336 & 11.0906 \\
\hline $\mathbf{C}$ & 5.12668 & 12.432 & 11.2447 \\
\hline $\mathbf{H}$ & 5.29979 & 12.1425 & 10.2083 \\
\hline $\mathbf{C}$ & 4.25358 & 11.6436 & 12.055 \\
\hline $\mathbf{C}$ & 3.57685 & 10.4631 & 11.5653 \\
\hline $\mathbf{C}$ & 3.79365 & 9.949 & 10.1767 \\
\hline $\mathbf{C}$ & 5.10726 & 9.59367 & 9.69215 \\
\hline $\mathbf{C}$ & 6.25628 & 9.5422 & 10.5384 \\
\hline $\mathbf{H}$ & 6.14052 & 9.75224 & 11.6026 \\
\hline $\mathbf{C}$ & 7.5074 & 9.22482 & 10.0437 \\
\hline $\mathbf{H}$ & 8.36481 & 9.19025 & 10.7217 \\
\hline $\mathbf{C}$ & 7.69076 & 8.93618 & 8.66827 \\
\hline $\mathbf{H}$ & 8.68426 & 8.69032 & 8.28348 \\
\hline $\mathbf{C}$ & 6.59823 & 8.93797 & 7.82577 \\
\hline $\mathbf{H}$ & 6.71313 & 8.68305 & 6.76795 \\
\hline & & & \\
\hline & & & \\
\hline $\mathbf{H}$ & & \\
\hline $\mathbf{H}$ & & \\
\hline
\end{tabular}




\begin{tabular}{cccc}
\hline $\mathbf{C}$ & 5.29473 & 9.24472 & 8.30899 \\
\hline $\mathbf{C}$ & 4.16132 & 9.19107 & 7.45059 \\
\hline $\mathbf{H}$ & 4.30377 & 8.92046 & 6.40048 \\
\hline $\mathbf{C}$ & 2.90254 & 9.43683 & 7.94123 \\
\hline $\mathbf{H}$ & 2.01953 & 9.36012 & 7.30319 \\
\hline $\mathbf{C}$ & 2.70469 & 9.80229 & 9.30508 \\
\hline $\mathbf{O}$ & -0.5089 & 10.4302 & 11.9686 \\
\hline $\mathbf{O}$ & -1.2982 & 9.42555 & 9.70255 \\
\hline $\mathbf{C}$ & -1.7757 & 10.5883 & 12.4059 \\
\hline $\mathbf{C}$ & -2.0326 & 10.3014 & 13.7777 \\
\hline $\mathbf{H}$ & -1.1816 & 10.0412 & 14.4109 \\
\hline $\mathbf{C}$ & -3.3106 & 10.3401 & 14.2795 \\
\hline $\mathbf{H}$ & -3.4994 & 10.1189 & 15.334 \\
\hline $\mathbf{C}$ & -4.4121 & 10.6181 & 13.4231 \\
\hline $\mathbf{C}$ & -5.7508 & 10.5694 & 13.9056 \\
\hline $\mathbf{H}$ & -5.9151 & 10.3419 & 14.9632 \\
\hline $\mathbf{C}$ & -6.8209 & 10.7732 & 13.0594 \\
\hline $\mathbf{H}$ & -7.8443 & 10.7224 & 13.4417 \\
\hline $\mathbf{C}$ & -6.585 & 11.0304 & 11.6858 \\
\hline $\mathbf{H}$ & -7.4319 & 11.1666 & 11.008 \\
\hline $\mathbf{C}$ & -5.2963 & 11.1084 & 11.1934 \\
\hline $\mathbf{H}$ & -5.1384 & 11.2981 & 10.1304 \\
\hline $\mathbf{C}$ & -4.1622 & 10.9259 & 12.04 \\
\hline $\mathbf{C}$ & -2.8079 & 10.9894 & 11.5458 \\
\hline $\mathbf{C}$ & -2.4871 & 11.4505 & 10.1577 \\
\hline $\mathbf{C}$ & -2.8708 & 12.7594 & 9.68129 \\
\hline $\mathbf{C}$ & -3.5608 & 13.7103 & 10.4932 \\
\hline $\mathbf{H}$ & -3.802 & 13.4557 & 11.5251 \\
\hline $\mathbf{C}$ & -3.9323 & 14.947 & 10.0012 \\
\hline $\mathbf{H}$ & -4.4627 & 15.6493 & 10.6507 \\
\hline $\mathbf{C}$ & -3.6257 & 15.32 & 8.66871 \\
\hline $\mathbf{H}$ & -3.9326 & 16.298 & 8.2887 \\
\hline & -2.9264 & 14.4441 & 7.86437 \\
\hline $\mathbf{C}$ & & & \\
\hline $\mathbf{H}$ & & & \\
\hline $\mathbf{H}$ & & \\
\hline
\end{tabular}




\begin{tabular}{cccc}
\hline $\mathbf{H}$ & -2.6515 & 14.7252 & 6.84343 \\
\hline $\mathbf{C}$ & -2.5321 & 13.1613 & 8.34041 \\
\hline $\mathbf{C}$ & -1.7923 & 12.2711 & 7.51138 \\
\hline $\mathbf{H}$ & -1.5242 & 12.5853 & 6.49892 \\
\hline $\mathbf{C}$ & -1.4035 & 11.042 & 7.98242 \\
\hline $\mathbf{H}$ & -0.8239 & 10.3505 & 7.36703 \\
\hline $\mathbf{C}$ & -1.741 & 10.6301 & 9.30368 \\
\hline $\mathbf{O}$ & -0.7476 & 7.65529 & 12.0844 \\
\hline $\mathbf{O}$ & 0.46414 & 7.24725 & 9.82245 \\
\hline $\mathbf{C}$ & -0.4441 & 6.41466 & 12.5083 \\
\hline $\mathbf{C}$ & -0.0232 & 6.27762 & 13.8636 \\
\hline $\mathbf{H}$ & 0.01086 & 7.18032 & 14.477 \\
\hline $\mathbf{C}$ & 0.32554 & 5.0519 & 14.374 \\
\hline $\mathbf{H}$ & 0.64054 & 4.9547 & 15.4166 \\
\hline $\mathbf{C}$ & 0.31912 & 3.89499 & 13.5453 \\
\hline $\mathbf{C}$ & 0.72667 & 2.62831 & 14.0507 \\
\hline $\mathbf{H}$ & 1.02296 & 2.56011 & 15.1016 \\
\hline $\mathbf{C}$ & 0.77174 & 1.51477 & 13.2373 \\
\hline $\mathbf{H}$ & 1.09388 & 0.55021 & 13.6389 \\
\hline $\mathbf{C}$ & 0.42667 & 1.6407 & 11.8682 \\
\hline $\mathbf{H}$ & 0.49296 & 0.76833 & 11.2119 \\
\hline $\mathbf{C}$ & 0.01487 & 2.85497 & 11.352 \\
\hline $\mathbf{H}$ & -0.2371 & 2.92749 & 10.2934 \\
\hline $\mathbf{C}$ & -0.082 & 4.02226 & 12.1694 \\
\hline $\mathbf{C}$ & -0.5319 & 5.2981 & 11.6639 \\
\hline $\mathbf{C}$ & -1.0705 & 5.45621 & 10.2769 \\
\hline $\mathbf{C}$ & -2.2121 & 4.70215 & 9.80972 \\
\hline $\mathbf{C}$ & -2.9423 & 3.80019 & 10.6427 \\
\hline $\mathbf{H}$ & -2.6319 & 3.65997 & 11.6782 \\
\hline $\mathbf{C}$ & -4.0376 & 3.10207 & 10.1704 \\
\hline $\mathbf{H}$ & -4.5724 & 2.42225 & 10.8396 \\
\hline $\mathbf{C}$ & -4.4817 & 3.26691 & 8.83485 \\
\hline $\mathbf{H}$ & -5.3498 & 2.7103 & 8.47177 \\
\hline & & & \\
\hline
\end{tabular}




\begin{tabular}{cccc}
\hline $\mathbf{C}$ & -3.8183 & 4.14856 & 8.00617 \\
\hline $\mathbf{H}$ & -4.162 & 4.31358 & 6.98067 \\
\hline $\mathbf{C}$ & -2.6863 & 4.88118 & 8.46238 \\
\hline $\mathbf{C}$ & -2.0261 & 5.80991 & 7.60916 \\
\hline $\mathbf{H}$ & -2.3886 & 5.9435 & 6.58581 \\
\hline $\mathbf{C}$ & -0.9694 & 6.552 & 8.07341 \\
\hline $\mathbf{H}$ & -0.4674 & 7.28779 & 7.44237 \\
\hline $\mathbf{C}$ & -0.509 & 6.40383 & 9.41261 \\
\hline
\end{tabular}


Table S11. XYZ coordinates and Gibbs free energy for the DFT structure of [1-Ta]' $E_{h}=-2813.39552574209$

\begin{tabular}{cccc}
\hline Atom & $\mathbf{X}$ & $\mathbf{Y}$ & $\mathbf{Z}$ \\
\hline $\mathbf{T a}$ & -0.1537 & 8.70394 & 10.8016 \\
\hline $\mathbf{O}$ & 1.58586 & 8.49593 & 11.9307 \\
\hline $\mathbf{O}$ & 1.04008 & 9.86779 & 9.59209 \\
\hline $\mathbf{C}$ & 2.25168 & 9.56731 & 12.3643 \\
\hline $\mathbf{C}$ & 2.03042 & 9.96829 & 13.7206 \\
\hline $\mathbf{H}$ & 1.36728 & 9.34084 & 14.3198 \\
\hline $\mathbf{C}$ & 2.60006 & 11.1025 & 14.2384 \\
\hline $\mathbf{H}$ & 2.39826 & 11.403 & 15.2712 \\
\hline $\mathbf{C}$ & 3.43603 & 11.9243 & 13.4289 \\
\hline $\mathbf{C}$ & 3.999 & 13.1274 & 13.9392 \\
\hline $\mathbf{H}$ & 3.76653 & 13.4105 & 14.9708 \\
\hline $\mathbf{C}$ & 4.80193 & 13.9327 & 13.1554 \\
\hline $\mathbf{H}$ & 5.23224 & 14.8523 & 13.5613 \\
\hline $\mathbf{C}$ & 5.05007 & 13.5613 & 11.8105 \\
\hline $\mathbf{H}$ & 5.66653 & 14.2059 & 11.1768 \\
\hline $\mathbf{C}$ & 4.51026 & 12.4021 & 11.2842 \\
\hline $\mathbf{H}$ & 4.71011 & 12.1487 & 10.2428 \\
\hline $\mathbf{C}$ & 3.69783 & 11.5257 & 12.0692 \\
\hline $\mathbf{C}$ & 3.12138 & 10.3096 & 11.5445 \\
\hline $\mathbf{C}$ & 3.38596 & 9.86011 & 10.1418 \\
\hline $\mathbf{C}$ & 4.72227 & 9.57612 & 9.68134 \\
\hline $\mathbf{C}$ & 5.85407 & 9.56351 & 10.5543 \\
\hline $\mathbf{H}$ & 5.70006 & 9.74857 & 11.6187 \\
\hline $\mathbf{C}$ & 7.13158 & 9.31676 & 10.0872 \\
\hline $\mathbf{H}$ & 7.97218 & 9.31294 & 10.7872 \\
\hline $\mathbf{C}$ & 7.36439 & 9.06368 & 8.7121 \\
\hline $\mathbf{H}$ & 8.37841 & 8.8756 & 8.34836 \\
\hline $\mathbf{C}$ & 6.29287 & 9.03189 & 7.84201 \\
\hline $\mathbf{H}$ & 6.44782 & 8.80852 & 6.78163 \\
\hline & & & \\
\hline
\end{tabular}




\begin{tabular}{cccc}
\hline $\mathbf{C}$ & 4.96459 & 9.26647 & 8.29563 \\
\hline $\mathbf{C}$ & 3.85488 & 9.18914 & 7.40754 \\
\hline $\mathbf{H}$ & 4.03487 & 8.95234 & 6.35427 \\
\hline $\mathbf{C}$ & 2.57673 & 9.38078 & 7.87077 \\
\hline $\mathbf{H}$ & 1.71363 & 9.29802 & 7.20552 \\
\hline $\mathbf{C}$ & 2.31298 & 9.70384 & 9.24072 \\
\hline $\mathbf{O}$ & -0.7134 & 10.1355 & 12.118 \\
\hline $\mathbf{O}$ & -1.9655 & 9.23339 & 9.86346 \\
\hline $\mathbf{C}$ & -1.9035 & 10.5411 & 12.5516 \\
\hline $\mathbf{C}$ & -2.2453 & 10.2364 & 13.9062 \\
\hline $\mathbf{H}$ & -1.5061 & 9.69033 & 14.4975 \\
\hline $\mathbf{C}$ & -3.453 & 10.6116 & 14.4403 \\
\hline $\mathbf{H}$ & -3.7036 & 10.3611 & 15.4755 \\
\hline $\mathbf{C}$ & -4.4024 & 11.3203 & 13.6505 \\
\hline $\mathbf{C}$ & -5.6599 & 11.7126 & 14.1918 \\
\hline $\mathbf{H}$ & -5.8785 & 11.457 & 15.2334 \\
\hline $\mathbf{C}$ & -6.5897 & 12.388 & 13.4274 \\
\hline $\mathbf{H}$ & -7.5473 & 12.6941 & 13.8575 \\
\hline $\mathbf{C}$ & -6.2956 & 12.6713 & 12.07 \\
\hline $\mathbf{H}$ & -7.0379 & 13.1854 & 11.4522 \\
\hline $\mathbf{C}$ & -5.0856 & 12.299 & 11.5151 \\
\hline $\mathbf{H}$ & -4.8918 & 12.5247 & 10.4665 \\
\hline $\mathbf{C}$ & -4.0796 & 11.6306 & 12.2806 \\
\hline $\mathbf{C}$ & -2.7982 & 11.2518 & 11.7317 \\
\hline $\mathbf{C}$ & -2.451 & 11.5427 & 10.3041 \\
\hline $\mathbf{C}$ & -2.4301 & 12.8865 & 9.78549 \\
\hline $\mathbf{C}$ & -2.5142 & 14.0385 & 10.6268 \\
\hline $\mathbf{H}$ & -2.5888 & 13.8963 & 11.707 \\
\hline $\mathbf{C}$ & -2.4847 & 15.3205 & 10.1101 \\
\hline $\mathbf{H}$ & -2.5404 & 16.1782 & 10.7866 \\
\hline $\mathbf{C}$ & -2.379 & 15.536 & 8.71291 \\
\hline $\mathbf{H}$ & -2.3659 & 16.553 & 8.31083 \\
\hline $\mathbf{C}$ & -2.2782 & 14.449 & 7.86751 \\
\hline & & & \\
\hline
\end{tabular}




\begin{tabular}{cccc}
\hline H & -2.1757 & 14.5956 & 6.78725 \\
\hline $\mathbf{C}$ & -2.2816 & 13.1183 & 8.3716 \\
\hline $\mathbf{C}$ & -2.1223 & 11.9975 & 7.50801 \\
\hline $\mathbf{H}$ & -2.0343 & 12.1675 & 6.43005 \\
\hline $\mathbf{C}$ & -2.0373 & 10.728 & 8.02407 \\
\hline $\mathbf{H}$ & -1.8664 & 9.86405 & 7.3762 \\
\hline $\mathbf{C}$ & -2.1497 & 10.4758 & 9.42964 \\
\hline $\mathbf{O}$ & -1.3551 & 7.31296 & 11.846 \\
\hline $\mathbf{O}$ & 0.13512 & 7.10335 & 9.56858 \\
\hline $\mathbf{C}$ & -0.7695 & 6.21367 & 12.3124 \\
\hline $\mathbf{C}$ & -0.3486 & 6.19104 & 13.6816 \\
\hline $\mathbf{H}$ & -0.5679 & 7.07531 & 14.2845 \\
\hline $\mathbf{C}$ & 0.31371 & 5.11371 & 14.2141 \\
\hline $\mathbf{H}$ & 0.63682 & 5.12177 & 15.2597 \\
\hline $\mathbf{C}$ & 0.62678 & 3.98324 & 13.4059 \\
\hline $\mathbf{C}$ & 1.36303 & 2.88284 & 13.9287 \\
\hline $\mathbf{H}$ & 1.67403 & 2.91722 & 14.9776 \\
\hline $\mathbf{C}$ & 1.70204 & 1.80332 & 13.1369 \\
\hline $\mathbf{H}$ & 2.27263 & 0.96774 & 13.552 \\
\hline $\mathbf{C}$ & 1.31666 & 1.7984 & 11.772 \\
\hline $\mathbf{H}$ & 1.60474 & 0.95996 & 11.1311 \\
\hline $\mathbf{C}$ & 0.59133 & 2.84815 & 11.2386 \\
\hline $\mathbf{H}$ & 0.31386 & 2.8244 & 10.1835 \\
\hline $\mathbf{C}$ & 0.20133 & 3.97372 & 12.0294 \\
\hline $\mathbf{C}$ & -0.5477 & 5.08478 & 11.4975 \\
\hline $\mathbf{C}$ & -1.0718 & 5.0913 & 10.095 \\
\hline $\mathbf{C}$ & -2.0245 & 4.108 & 9.63726 \\
\hline $\mathbf{C}$ & -2.5719 & 3.10098 & 10.4925 \\
\hline $\mathbf{H}$ & -2.2581 & 3.06619 & 11.5365 \\
\hline $\mathbf{C}$ & -3.4917 & 2.17606 & 10.0342 \\
\hline $\mathbf{H}$ & -3.8904 & 1.42602 & 10.7233 \\
\hline $\mathbf{C}$ & -3.9299 & 2.19547 & 8.68555 \\
\hline $\mathbf{H}$ & -4.6574 & 1.4595 & 8.33176 \\
\hline & & & \\
\hline & & & \\
\hline
\end{tabular}




\begin{tabular}{cccc}
\hline $\mathbf{C}$ & -3.4368 & 3.1613 & 7.83122 \\
\hline $\mathbf{H}$ & -3.7761 & 3.20504 & 6.79164 \\
\hline $\mathbf{C}$ & -2.4921 & 4.13037 & 8.27467 \\
\hline $\mathbf{C}$ & -2.0073 & 5.14111 & 7.39746 \\
\hline $\mathbf{H}$ & -2.3605 & 5.15766 & 6.36189 \\
\hline $\mathbf{C}$ & -1.1279 & 6.09393 & 7.84951 \\
\hline $\mathbf{H}$ & -0.7587 & 6.88425 & 7.1915 \\
\hline $\mathbf{C}$ & -0.6709 & 6.10551 & 9.20451 \\
\hline
\end{tabular}


Table S12. XYZ coordinates and Gibbs free energy for the DFT structure of [1-Ta]+. $E_{h}=-2813.395525742097$

\begin{tabular}{cccc}
\hline Atom & $\mathbf{X}$ & $\mathbf{Y}$ & $\mathbf{Z}$ \\
\hline $\mathbf{T a}$ & 0.35592 & 9.02772 & 10.8597 \\
\hline $\mathbf{O}$ & 1.9951 & 8.88655 & 11.9658 \\
\hline $\mathbf{O}$ & 1.54613 & 10.0803 & 9.69776 \\
\hline $\mathbf{C}$ & 2.79378 & 9.8875 & 12.4068 \\
\hline $\mathbf{C}$ & 2.61689 & 10.2995 & 13.7572 \\
\hline $\mathbf{H}$ & 1.90909 & 9.74107 & 14.3731 \\
\hline $\mathbf{C}$ & 3.30331 & 11.379 & 14.2532 \\
\hline $\mathbf{H}$ & 3.14916 & 11.7048 & 15.2857 \\
\hline $\mathbf{C}$ & 4.20053 & 12.1074 & 13.422 \\
\hline $\mathbf{C}$ & 4.87753 & 13.2583 & 13.9152 \\
\hline $\mathbf{H}$ & 4.68957 & 13.5654 & 14.9483 \\
\hline $\mathbf{C}$ & 5.7337 & 13.9827 & 13.1114 \\
\hline $\mathbf{H}$ & 6.25045 & 14.8627 & 13.5031 \\
\hline $\mathbf{C}$ & 5.92475 & 13.5848 & 11.7651 \\
\hline $\mathbf{H}$ & 6.58375 & 14.1686 & 11.1164 \\
\hline $\mathbf{C}$ & 5.27788 & 12.4745 & 11.2559 \\
\hline $\mathbf{H}$ & 5.43668 & 12.2019 & 10.2127 \\
\hline $\mathbf{C}$ & 4.4077 & 11.6806 & 12.063 \\
\hline $\mathbf{C}$ & 3.71191 & 10.5162 & 11.5604 \\
\hline $\mathbf{C}$ & 3.91245 & 10.022 & 10.1616 \\
\hline $\mathbf{C}$ & 5.22205 & 9.66405 & 9.66521 \\
\hline $\mathbf{C}$ & 6.37206 & 9.58809 & 10.5075 \\
\hline $\mathbf{H}$ & 6.26153 & 9.7808 & 11.5754 \\
\hline $\mathbf{C}$ & 7.61816 & 9.26638 & 10.0033 \\
\hline $\mathbf{H}$ & 8.47719 & 9.21132 & 10.6777 \\
\hline $\mathbf{C}$ & 7.79392 & 8.99811 & 8.62286 \\
\hline $\mathbf{H}$ & 8.78369 & 8.74839 & 8.23129 \\
\hline $\mathbf{C}$ & 6.6996 & 9.02412 & 7.78349 \\
\hline $\mathbf{H}$ & 6.80877 & 8.78486 & 6.72154 \\
\hline & & & \\
\hline $\mathbf{H}$ & & \\
\hline
\end{tabular}




\begin{tabular}{cccc}
\hline $\mathbf{C}$ & 5.40083 & 9.33641 & 8.2761 \\
\hline $\mathbf{C}$ & 4.2653 & 9.30777 & 7.41967 \\
\hline $\mathbf{H}$ & 4.40201 & 9.05528 & 6.36451 \\
\hline $\mathbf{C}$ & 3.01002 & 9.5548 & 7.91858 \\
\hline $\mathbf{H}$ & 2.12397 & 9.49846 & 7.28263 \\
\hline $\mathbf{C}$ & 2.82503 & 9.89448 & 9.28859 \\
\hline $\mathbf{O}$ & -0.4078 & 10.5056 & 11.9177 \\
\hline $\mathbf{O}$ & -1.1666 & 9.43426 & 9.67318 \\
\hline $\mathbf{C}$ & -1.673 & 10.6308 & 12.3918 \\
\hline $\mathbf{C}$ & -1.8581 & 10.4202 & 13.7864 \\
\hline $\mathbf{H}$ & -0.9699 & 10.2529 & 14.3994 \\
\hline $\mathbf{C}$ & -3.1167 & 10.4231 & 14.3351 \\
\hline $\mathbf{H}$ & -3.2543 & 10.2682 & 15.4091 \\
\hline $\mathbf{C}$ & -4.2615 & 10.5764 & 13.5047 \\
\hline $\mathbf{C}$ & -5.5759 & 10.4821 & 14.043 \\
\hline $\mathbf{H}$ & -5.6866 & 10.3182 & 15.1193 \\
\hline $\mathbf{C}$ & -6.6869 & 10.5671 & 13.23 \\
\hline $\mathbf{H}$ & -7.6902 & 10.4826 & 13.6563 \\
\hline $\mathbf{C}$ & -6.5175 & 10.7419 & 11.8338 \\
\hline $\mathbf{H}$ & -7.3955 & 10.7784 & 11.1833 \\
\hline $\mathbf{C}$ & -5.2546 & 10.8615 & 11.2857 \\
\hline $\mathbf{H}$ & -5.1482 & 10.9858 & 10.2068 \\
\hline $\mathbf{C}$ & -4.0813 & 10.8067 & 12.0961 \\
\hline $\mathbf{C}$ & -2.7503 & 10.9268 & 11.5465 \\
\hline $\mathbf{C}$ & -2.5119 & 11.3623 & 10.1329 \\
\hline $\mathbf{C}$ & -3.0229 & 12.6203 & 9.63377 \\
\hline $\mathbf{C}$ & -3.7536 & 13.54 & 10.4457 \\
\hline $\mathbf{H}$ & -3.9277 & 13.3054 & 11.4954 \\
\hline $\mathbf{C}$ & -4.2508 & 14.7223 & 9.93151 \\
\hline $\mathbf{H}$ & -4.8104 & 15.4 & 10.5825 \\
\hline $\mathbf{C}$ & -4.0328 & 15.0736 & 8.5764 \\
\hline $\mathbf{C}$ & -4.4343 & 16.01 & 8.18039 \\
\hline & -3.2935 & 14.2325 & 7.77058 \\
\hline & & & \\
\hline $\mathbf{H}$ & & \\
\hline $\mathbf{H}$ & & \\
\hline
\end{tabular}




\begin{tabular}{cccc}
\hline H & -3.0806 & 14.5017 & 6.73177 \\
\hline $\mathbf{C}$ & -2.7749 & 13.0038 & 8.2681 \\
\hline $\mathbf{C}$ & -1.9998 & 12.1513 & 7.43168 \\
\hline $\mathbf{H}$ & -1.801 & 12.4535 & 6.39993 \\
\hline $\mathbf{C}$ & -1.4916 & 10.9746 & 7.91981 \\
\hline $\mathbf{H}$ & -0.8848 & 10.3111 & 7.29959 \\
\hline $\mathbf{C}$ & -1.7361 & 10.5837 & 9.26646 \\
\hline $\mathbf{O}$ & -0.6297 & 7.74159 & 12.129 \\
\hline $\mathbf{O}$ & 0.54687 & 7.2915 & 9.8559 \\
\hline $\mathbf{C}$ & -0.4576 & 6.51644 & 12.5452 \\
\hline $\mathbf{C}$ & -0.2445 & 6.3154 & 13.9416 \\
\hline $\mathbf{H}$ & -0.2306 & 7.20166 & 14.578 \\
\hline $\mathbf{C}$ & -0.0707 & 5.05865 & 14.4591 \\
\hline $\mathbf{H}$ & 0.0918 & 4.92452 & 15.5321 \\
\hline $\mathbf{C}$ & -0.052 & 3.90269 & 13.6141 \\
\hline $\mathbf{C}$ & 0.20988 & 2.62153 & 14.1465 \\
\hline $\mathbf{H}$ & 0.36207 & 2.51931 & 15.2244 \\
\hline $\mathbf{C}$ & 0.29653 & 1.50912 & 13.3164 \\
\hline $\mathbf{H}$ & 0.50118 & 0.5237 & 13.742 \\
\hline $\mathbf{C}$ & 0.15703 & 1.66814 & 11.9251 \\
\hline $\mathbf{H}$ & 0.26824 & 0.80485 & 11.2646 \\
\hline $\mathbf{C}$ & -0.1082 & 2.91906 & 11.381 \\
\hline $\mathbf{H}$ & -0.1865 & 3.02353 & 10.298 \\
\hline $\mathbf{C}$ & -0.2654 & 4.06404 & 12.2066 \\
\hline $\mathbf{C}$ & -0.5644 & 5.37409 & 11.6641 \\
\hline $\mathbf{C}$ & -1.0537 & 5.54834 & 10.2949 \\
\hline $\mathbf{C}$ & -2.1779 & 4.78305 & 9.77355 \\
\hline $\mathbf{C}$ & -2.9936 & 3.94933 & 10.5858 \\
\hline $\mathbf{H}$ & -2.7721 & 3.84032 & 11.6473 \\
\hline $\mathbf{C}$ & -4.0831 & 3.26917 & 10.0613 \\
\hline $\mathbf{H}$ & -4.6848 & 2.63523 & 10.7176 \\
\hline $\mathbf{C}$ & -4.4306 & 3.40045 & 8.70085 \\
\hline & -5.2924 & 2.86336 & 8.29726 \\
\hline $\mathbf{H}$ & & & \\
\hline $\mathbf{H}$ & & \\
\hline $\mathbf{H}$ & & \\
\hline
\end{tabular}




\begin{tabular}{llll}
\hline $\mathbf{C}$ & -3.6813 & 4.23569 & 7.88842 \\
\hline $\mathbf{H}$ & -3.9566 & 4.38655 & 6.84106 \\
\hline $\mathbf{C}$ & -2.5593 & 4.9352 & 8.39917 \\
\hline $\mathbf{C}$ & -1.8228 & 5.82585 & 7.56139 \\
\hline $\mathbf{H}$ & -2.1168 & 5.92616 & 6.51291 \\
\hline $\mathbf{C}$ & -0.7865 & 6.57577 & 8.05903 \\
\hline $\mathbf{H}$ & -0.2286 & 7.2689 & 7.42793 \\
\hline $\mathbf{C}$ & -0.4228 & 6.49184 & 9.42831 \\
\hline
\end{tabular}




\section{References}

1. CrysAlisPro 1.171.40.81a: Rigaku Oxford Diffraction; 2019, Rigaku Corporation, Oxford, UK. 2. SCALE3 ABSPACK v1.0.7: an Oxford Diffraction program; 2005, Oxford Diffraction Ltd: Abingdon, UK.

3. Sheldrick G. SHELXT - Integrated space-group and crystal-structure determination. Acta Cryst. A 2015, 71, 3-8. 